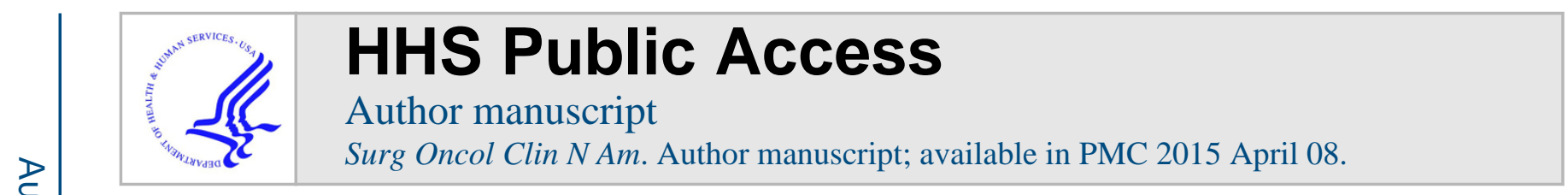

Published in final edited form as:

Surg Oncol Clin N Am. 2008 January ; 17(1): 93-viii. doi:10.1016/j.soc.2007.10.014.

\title{
Surgery for Thyroid Cancer
}

\author{
Ziv Gil, MD, PhD and Snehal G. Patel, MD* \\ Head and Neck Service, Department of Surgery, Memorial Sloan-Kettering Cancer Center, 1275 \\ York Avenue, New York, NY 10065, USA
}

Surgical resection of the thyroid gland is standard treatment for management of carcinomas and is performed by a variety of specialists, including general surgeons, otolaryngologists, endocrine surgeons, and oncologic surgeons. Oncologic thyroid surgery has three main objectives: to assure complete removal of one or both of the thyroid lobes, to preserve the parathyroid (PT) glands and their blood supply, and to prevent injury to the superior and recurrent laryngeal nerves (RLNs). To perform safe and efficient thyroid surgery, surgeons should be familiar not only with the anatomy of the thyroid gland and the vital structures along the course of the gland but also with the anatomic variations in regional anatomy. Although postoperative complications are infrequent in experienced hands, hypocalcemia and vocal cord paralysis can lead to significant morbidity and impaired quality of life. The operation of thyroidectomy has been performed for more than a century [1] and is well described in the literature with the expected variation in the actual technique of the procedure.

This article describes the technique of thyroidectomy in detail as practiced by the authors at Memorial Sloan-Kettering Cancer Center for the surgical treatment of thyroid cancer. A technique that facilitates complete extracapsular resection of the thyroid, preserves the PT glands with their blood supply, the RLNs, and the superior laryngeal nerves, and minimizes postoperative complications is emphasized. Management of certain specific situations, such as retrosternal goiter, locally invasive thyroid cancer, and reoperative surgery for locally recurrent cancer, is discussed but management of the regional lymph nodes is not addressed here.

\section{Standard surgical technique of thyroidectomy}

The extent of surgical resection of the thyroid gland for well-differentiated thyroid cancer (WDTC) has been debated extensively and the indications and pros and cons of hemiversus total thyroidectomy are discussed in the article by Witt elsewhere in this issue. The authors practice a policy of risk stratification in deciding the extent of surgery, and the

() 2008 Elsevier Inc. All rights reserved.

"Corresponding author. patels@mskcc.org (S.G. Patel).

Publisher's Disclaimer: This is a PDF file of an unedited manuscript that has been accepted for publication. As a service to our customers we are providing this early version of the manuscript. The manuscript will undergo copyediting, typesetting, and review of the resulting proof before it is published in its final citable form. Please note that during the production process errors may be discovered which could affect the content, and all legal disclaimers that apply to the journal pertain. 
technique of unilateral extirpation of the thyroid lobe is described, as bilateral resection is performed in a similar fashion.

\section{Skin incision planning}

Skin incision preferably should be marked with patients awake and sitting up. The skin incision is planned so that it provides optimal surgical exposure for safe conduct of the operation and results in the best cosmetic outcome. A suitable transverse line of relaxed skin tension in the lower neck most commonly fulfills these requirements.

Variability in body habitus and the extent of access required dictate the location and length of the skin incision, and the site and length of the incision never should be based on cosmetic considerations alone. Planning of the incision should take into account the length and width of the patient's neck, the size of the thyroid gland, and presence of previous surgical scars (Fig. 1). Patients who have a long neck may require selection of a higher skin crease to allow full access to the superior poles of the thyroid gland, which are located more cephalad than in patients who have a short neck. If the usual lower skin crease is chosen in patients who have a long neck, a longer incision is required in order to provide sufficient access to the superior poles. Careful planning of the incision in female patients who have large breasts is especially important, because the skin over the manubrium tends to ride up into the neck when the patient is supine on the operating table. A higher skin crease may need to be selected in these patients in order to avoid a wide, hypertrophic scar and poor cosmetic outcome is seen not uncommonly in incisions that come to lie on the skin of the upper chest.

An incision that extends from the anterior border of one sternocleido-mastoid muscle to the other generally is adequate for routine thyroidectomy. This incision usually is sufficient to provide access for paratracheal node dissection, but lateral extension along the skin crease to the anterior border of the trapezius muscle is required for access to levels II-V in the lateral neck. Lateral extension of the incision also may be required for delivery of a large goiter.

\section{Positioning and draping}

The patient is placed on the operating room table in a supine position. General anesthesia is administered and an oral endotracheal tube inserted by an anesthesiologist. Unless there are specific medical requirements, a 6- to $6.5-\mathrm{mm}$ size endotracheal tube is used routinely in order to minimize trauma to the vocal cords. Lacrilube ophthalmic ointment is applied to both eyes before they are taped shut with Microfoam tape. Venodyne pressure devices are used routinely, although deep vein thrombosis is uncommon after thyroid surgery. The head is stabilized with a soft donut holder and the operating table is elevated at a $30^{\circ}$ angle with the head up (reverse Trendelenburg) and the neck in extension (Fig. 2). Exceptions may need to be made for patients who have restricted mobility of the spine and special care must be taken to avoid injury. Adequate extension of the neck may not be attained in obese patients unless folded sheets are placed under the shoulders to elevate them.

Severe neuropraxia of the brachial plexus is prevented by avoiding stretching of the arms. Injury to the ulnar nerves is avoided by protecting the elbows with adequate padding. 
Hypotensive anesthesia is preferable for minimizing bleeding, and communication with the anesthesiologist is crucial in this regard. Prophylactic antibiotics have been shown ineffective in reducing infections after thyroid surgery [2] and the authors do not administer them routinely.

The surgeon should be aware of the possibility that extension of the neck may pull the endotracheal tube out 2 to $4 \mathrm{~cm}$ from its original location. Caudal repositioning of the tube may be required, and the position of the tube should always be confirmed before prepping and draping. The patient is prepped and draped in a standard surgical fashion. A transparent plastic drape is used to drape the head (from the level of the jaw line upward) to allow continuous monitoring of the endotracheal tube and anesthesia circuit (see Fig. 2).

\section{Development of cutaneous flaps}

The skin is incised with a \#15 blade and subsequent dissection is performed with electrocautery at the lowest effective setting. The platysma muscle is incised and cutaneous flaps are developed in the subplatysmal plane (Fig. 3). This plane separates the superficial cervical fascia from the superficial layer of the deep cervical fascia. Care is taken to prevent injury to the anterior jugular veins, which are maintained on the superficial layer of the deep cervical fascia. The superior flap is developed to a point approximately $1 \mathrm{~cm}$ cephalad to the thyroid notch and the inferior flap to the level of the superior border of the manubrium sterni. The flaps are retracted and held in place using elastic stay hooks or a Mahorner retractor.

\section{Exposure and management of the strap muscles}

The superficial layer of the deep cervical fascia is divided along the avascular midline between the infrahyoid strap muscles. Small veins interconnecting the anterior jugular veins are clamped and divided. The isthmus of the thyroid gland lies immediately deep in the midline and care is taken to avoid injury to the thyroid tissue and vessels during this part of the dissection. After incision of the midline fascia from the level of the cricoid to sternal notch, dissection and appropriate management of the infrahyoid strap muscles is the next step in exposure of the thyroid lobes, especially the superior poles.

A small thyroid gland can be exposed adequately without dividing the sternothyroid muscles (STMs). In this scenario, the strap muscles are retracted laterally and elevated meticulously off the thyroid capsule by dissecting in the avascular areolar tissue that separates the two structures, allowing full exposure of the thyroid lobe. Lateral retraction of the infrahyoid strap muscles almost always provides adequate exposure of the lower pole and body of the thyroid lobe. Adequate exposure of the superior pole and its anatomic relations, however, may be inadequate unless the STM is divided close to its attachment to the thyroid cartilage. Further division of the fascia between the sternohyoid muscle (SHM) and the medial border of the sternocleidomastoid muscle is a useful maneuver that allows wider retraction and better exposure of the superior pole of the thyroid without division of the STM. If lateral retraction of the strap muscles does not provide adequate exposure of the superior pole, the surgeon should have a low threshold for division of the STM. In such instances, the SHM is separated from the STM using the electrocautery. Bipolar electrocautery is used to coagulate 
blood vessels in the areolar tissue between the two strap muscles. Lateral retraction of the SHM while retracting the thyroid lobe medially allows exposure of the STM and its attachment to the thyroid cartilage. The internal jugular vein can be demonstrated at the lateral border of the STM. The superior attachment of the STM is identified clearly and the electrocautery is used to carefully divide the muscle close to this attachment. Division of the STM close to its superior attachment facilitates exposure of the superior thyroid artery and relevant anatomy and preserves muscle in-nervation by the ansa hypoglossus.

The divided STM then is retracted laterally for the remainder of the operation and no attempt is made to suture it back it into place. In selected instances, a segment of the STM may need to be resected in continuity with the underlying thyroid gland. Gross extrathyroidal extension (ETE) of tumor that is identified clinically, radiologically, or intraoperatively is an indication for such a maneuver. The STM also may be resected in order to provide an adequate surgical margin if ETE is suspected either pre- or intraoperatively. After division of the STM close to its superior attachment, the muscle is transected inferiorly at the level of the sternal notch so that the segment that abuts the tumor is left in situ on the thyroid lobe.

Although division of the STM is required not infrequently, the SHM only rarely needs to be divided in the absence of gross involvement by tumor. Exposure and delivery of a large goiter, especially with retrosternal extension, may be facilitated, however, by division of both infrahyoid strap muscles.

After exposure of the thyroid gland, both lobes are inspected carefully and palpated before proceeding further. If the contralateral lobe is normal on intraoperative examination and total thyroidectomy is indicated, the authors generally prefer to start with the normal side before dissecting the abnormal lobe. Apart from allowing safe dissection and preservation of the PT glands and RLN in the presence of undistorted normal regional anatomy, this approach provides the surgeon an indication of potential anatomic variations on the contralateral pathologic side.

\section{Exposure of the superior thyroid pole and preservation of the superior laryngeal nerve}

Dissection of the thyroid lobe begins by delineating the extent of its superior pole. If the STM is not divided, retraction of the strap muscles with a Richardson retractor laterally and with a right angle retractor cephalad, facilitates exposure of the tip the superior pole. The most important anatomic relation of the superior thyroid pedicle is the external branch of the superior laryngeal nerve (ESLN), but the surgeon also should be aware of the superior PT gland and its vascular supply during this dissection. Division of the superior thyroid vascular pedicle is an important initial step in mobilization of the thyroid lobe.

Mass ligature of the superior pedicle is ill advised for several reasons: (1) the extent of thyroid parenchymal tissue at the superior thyroid pole is variable and its cephalad-most portion easily can be left behind after thyroidectomy, causing superfluous uptake during postsurgical radioactive iodine (RAI) scanning; (2) the ESLN often accompanies the superior thyroid vessels and may be injured during mass ligation of the pedicle; (3) high ligation of the superior thyroid artery may compromise blood supply to the superior PT 
gland; (4) the superior PT gland occasionally is located posteriorly at the superior pole and inadvertently may be resected with the specimen; and (5) bleeding from a mass ligated superior pedicle may be difficult to control, because the vessels retract cephalad and the ESLN is at risk for injury if blind application of hemostats is attempted. Before ligation of the superior thyroid vessels, the surgeon should be aware of three anatomic variants of the main trunk of ESLN (Fig. 4). In the first variant, the ESLN descends superficial to the inferior constrictor muscle, traveling along the superior thyroid vessels before innervating the cricothyroid muscle. In the second variant, the ESLN pierces the inferior constrictor muscle approximately $1 \mathrm{~cm}$ above the cricothyroid membrane. In the third variant, the ESLN runs deep to the inferior constrictor muscle, thereby protecting the nerve from unintended injury [3]. High and mass ligation of the superior thyroid pedicle increases the risk for inadvertent injury to the ESLN in the first two anatomic variants, and the nerve would be safe only in the third variant where it runs deep to the fibers of the inferior constrictor muscle. For the reasons discussed previously, the authors prefer to ligate the individual branches of the superior thyroid artery and veins. These vessels frequently are found anterior to the medial aspect of the superior pole. The technique of individual vessel ligation allows the surgeon to delineate the thyroid parenchymal tissue at the superior pole from its surrounding structures (Fig. 5). Division of the anterior branches releases the tethered pole, exposing its superior and posterior borders. As the dissection continues along the posterior border of the superior pole, the pole gradually drops down caudally, away from the ESLN, and the remaining small posterior vessels can be cauterized safely with a finetipped bipolar electrocautery (Fig. 6). Separation of the superior pole from its superomedial attachment to the fascia surrounding the cricothyroid muscles often is required. Within this tissue, tiny vessels not infrequently interconnect the superior thyroid vessels to the fascia covering the cricothyroid muscles. These vessels should be cauterized carefully with a bipolar electrocautery close to the gland in order to prevent inadvertent injury to the ESLN.

\section{Capsular dissection of the thyroid lobe and preservation of the parathyroid glands}

After mobilization of the superior pole of the thyroid lobe, the next step is medial rotation of the lobe to provide exposure to its posterolateral surface. The middle thyroid vein, if present, is divided and ligated. Hemostatic clamps are applied carefully to the capsule of the thyroid lobe along its lateral edge. Gentle medial retraction of these clamps combined with lateral retraction of the strap muscles provides the surgical exposure required for identification of the PT glands and the RLN. As a general rule, the authors do not attempt to identify the RLN laterally in the paratracheal groove as the initial step. Instead, the focus of the dissection at this stage should be to identify and preserve the PT glands along with their vascular supply.

A technique of capsular dissection is recommended in order to prevent devascularization of the PT glands. The aim of this maneuver is to sweep the posterior capsular fascia off the thyroid parenchyma. This fascia consists of multiple thin layers of connective tissue that encapsulate the thyroid gland and its superficial vasculature. The PT glands frequently are embedded in this capsular tissue and receive their blood supply from branches of the inferior thyroid artery (ITA) and less frequently from the superior thyroid artery (Fig. 7). With the thyroid lobe rotated medially into the surgical wound, the areolar tissue on the posterior 
surface of the lobe is incised longitudinally and stripped away laterally from the thyroid. Small blood vessels encountered during this dissection are cauterized carefully with a finetipped bipolar forceps or ligated.

During lateral reflection of the visceral fascia, the terminal branches of the ITA are encountered distal to the PT gland after they have supplied these glands. These distal branches are divided carefully and ligated and the adventitial tissue around the thyroid capsule is peeled away along with the PT gland (Fig. 8). As described in the following section, further dissection during preservation of the inferior PT gland inevitably leads to identification of the RLN.

This technique allows identification and preservation of the superior and inferior PT glands along with their blood supply that is contained within the adventitial tissue surrounding the thyroid capsule. The PT glands can be distinguished from the underlying thyroid parenchyma by their yellowish brown color (café ole), fatty consistency, and teardrop shape. On a rare occasion, the surgeon may need to harvest a small sliver of an assumed PT gland for intraoperative pathologic examination to confirm the diagnosis. Devascularization of the PT gland leads to change in the color of the gland from light to dark brown within a few minutes. If a PT gland is detached from its blood supply, the authors recommend reimplantation of the gland after confirming its histology using intraoperative frozen section analysis. PT gland reimplantation is performed by mincing the devascularized gland and placing it into a small pocket created by blunt dissection of the fibers of the sternocleidomastoid muscle. The site of reimplantation is marked with nonabsorbable suture or small titanium clips in case the site needs to be identified at future surgery.

\section{Identification of the recurrent laryngeal nerve and its anatomic variations}

The authors' routine practice is to avoid dissection and identification of the RLN laterally in the paratracheal groove as the initial step. As described previously, capsular dissection during preservation of the inferior PT gland normally allows exposure of the RLN as it lies in relationship to the branches of the ITA. The authors prefer this approach rather than lateral dissection in order to avoid several pitfalls. First, lateral dissection for identification of the RLN away from the thyroid capsule increases the risk for devascularization of the PT glands. Second, blind dissection with a clamp along the suspected course of the RLN inadvertently may traumatize the nerve causing temporary or even permanent paralysis of the nerve. Finally, injury to blood vessels along the presumed course of the RLN may cause bleeding and attempts at hemostasis may place the as-yet unidentified nerve at risk for injury.

The alternative technique described here takes into consideration the normal anatomic relationship of the RLN to the ITA, the tuberculum Zuckerkandl, the trachea, and the esophagus.

The technique of capsular dissection is predicated on precise and meticulous surgical technique. Fine-tipped microclamps are used to spread alveolar tissue gently and clamp the distal branches of the ITA before division and ligation. Blind application of clamps and mass ligation of tissue is to be avoided at all costs. Identifiable blood vessels should be 
divided and ligated individually to prevent accidental injury to the RLN. As dissection of the branches of the ITA distal to the inferior PT gland nears completion, the RLN generally comes into view at this point. A small vasa nervosum vessel that accompanies the nerve can facilitate its identification and differentiation from other structures. The relationship of the RLN to the ITA and its branches, however, is variable and bears special attention (Fig. 9). In most individuals, the RLN is located deep to the ITA but it may lie between the branches or anterior to the artery [4]. The RLN generally courses deep to the tuberculum Zuckerkandl and superficial to the lateral border of the trachea. Variations in anatomic relationships of the RLN to the tuberculum Zuckerkandl are not infrequent, however, and the surgeon should be familiar with these in order to avoid injury to the RLN, especially when nodular pathology affects this part of the gland (Fig. 10). The relationship of the RLN to the tracheoesophageal groove also bears notice (Fig. 11). In more than half of cases, the RLN is located along the tracheoesophageal groove and passes deep to Berry's ligament (the posterior suspensory ligament of the thyroid). In the remaining cases, the RLN may pierce Berry's ligament or lie between the ligament and the thyroid parenchyma. The RLN also can ascend along the paraesophageal line posterior to the tracheoesophageal groove. Other variations in the location of the RLN are related to the side of the operated neck. In the left side of the neck, the RLN crosses anterior to the aortic arch, loops under it, and ascends in a relatively constant position along the tracheoesophageal groove (Fig. 12). The course of the right RLN is less predictable, because it crosses anterior to the subclavian artery and ascends posterior to the artery starting a point more lateral than in the left neck.

As the capsular dissection continues, the RLN is exposed laterally or medial to the tuberculum Zuckerkandl. After identification, the RLN is dissected gently away from the gland toward Berry's ligament. This dense condensation of the pretracheal fascia overlying the first and second tracheal rings "suspends" the thyroid lobe from the trachea. A finetipped hemostat is used to carefully spread the areolar tissue overlying the RLN. Small blood vessels encountered during the course of this dissection can be cauterized carefully with a fine-tipped bipolar forceps, but larger identifiable branches of the ITA must be clamped, divided, and ligated. The relationship of the RLN to Berry's ligament is determined and fine blood vessels in the ligament are clamped carefully with a fine-tipped microclamp before division with a scalpel and ligation. Special care must be exercised in placing this clamp in order to avoid inadvertent injury to the RLN. Similarly, the surgeon's finger or instrument should be oriented longitudinally along the course of the RLN so that the ligature does not exert pressure on the RLN while the knot is secured. The authors prefer using a needle driver to tie knots in this area, as this allows precise control and avoids inadvertent tearing of tissue and blood vessels. Bleeding from a loose or torn ligature on these vessels can be troublesome and it is important to avoid blind attempts at hemostasis with clamps. The safest course of action in this situation is to apply gentle pressure with a swab to achieve temporary hemostasis. This allows identification of the divided vessel and its safe ligation without placing the RLN at risk for injury. If the area of Berry's ligament continues to ooze, hemostasis can be achieved by placing a piece of hemostatic Surgicel instead of undue manipulation in attempts to place hemostatic ligatures. As it approaches the larynx, the nerve may run in a single bundle or may divide into 2 to 6 small branches before 
entering the cricothyroid junction. It is important to recognize this fact so that all branches of the RLN are preserved during dissection.

On rare occasions, a posteriorly located thyroid tumor may adhere to the perineurium of the RLN or may infiltrate deep into the perineural space in the region of Berry's ligament. Isolated invasion of the RLN without involvement of adjacent structures, such as the cricothyroid membrane, cricoid cartilage, tracheal rings, and esophageal musculature, is rare. If the RLN is paralyzed preoperatively and intraoperative exploration shows neural invasion by cancer, the RLN should be sacrificed in order to achieve gross total resection of the tumor. Decision making becomes difficult, however, in cases of minimal neural invasion by WDTC with normal RLN function. The decision to sacrifice a normally functioning RLN in patients who have WDTC is based on several factors, especially because WDTC generally is a RAI avid tumor so that microscopic residual tumor theoretically is amenable to RAI therapy. The primary intraoperative consideration in this decision is whether or not sacrifice of the functioning RLN would make the difference between total resection of the tumor with microscopically negative margins and an incomplete resection. Complete surgical resection of WDTC offers the best prospect for local control and every effort should be made to achieve gross total resection with microscopically negative surgical margins. Therefore, sacrifice of the RLN is indicated in the presence of gross invasion by tumor if this will achieve total tumor resection. Alternatively, the surgeon may be able to dissect a functioning RLN away from the tumor leaving microscopic disease on it. The functional consequences of RLN dysfunction need to be weighed against the likelihood of local recurrence if microscopic residual disease on the RLN fails to respond to RAI therapy. Older patients are more likely to have non-RAI-avid tumors; microscopic residual disease on the RLN is unlikely to respond to adjuvant RAI in this group. Resection of a functioning RLN becomes a valid consideration in these situations; yet, the functional consequences of RLN sacrifice are least likely to be tolerated by these same patients. If a tumor is adherent to the RLN and can be dissected away easily, a functioning RLN generally should be preserved. RLN sacrifice becomes necessary, however, for gross invasion of the nerve, especially in patients who have undifferentiated carcinoma that is unlikely to respond to RAI. Prior to sacrificing a functional nerve, it should be confirmed that the contralateral nerve is free of tumor and can be preserved safely. This is another reason for performing lobectomy on the contralateral side before approaching the diseased lobe or the side of the more prominent pathology.

Division of Berry's ligament frees the thyroid lobe from its attachment to the trachea. The inferior thyroid veins are divided and ligated close to the thyroid gland to complete mobilization of the lower pole of the lobe. In $10 \%$ of patients, a thyroid ima artery may be present anterior to the trachea. This artery emerges directly from the arch of the aorta or occasionally from the brachiocephalic trunk to supply the inferior pole of the gland and should be ligated at the level of the thyroid capsule.

Subsequent mobilization of the thyroid lobe off the anterolateral surface of the upper tracheal rings is accomplished with an electrocautery. Attention is given to identification and dissection of the pyramidal "lobe," which is found in more than 50\% of individuals $[5,6]$. The pyramidal lobe can branch off from the left or right thyroid lobes or from the isthmus. 
The lobe is dissected free from the underlying cricothyroid muscle using electrocautery in a superior-to-inferior direction to maintain it in continuity with the rest of the thyroid gland. If thyroid lobectomy is planned, the thyroid lobe and isthmus are dissected off the trachea and a Kocher clamp is placed vertically across the junction of the isthmus with the contralateral lobe. The thyroid parenchyma then is divided over the clamp with a \#15 scalpel to deliver the specimen. The cut edge of the remnant thyroid gland is oversewn with a continuous 3-0 chromic catgut suture. Alternatively, if total thyroidectomy is planned, lobectomy is performed on the contralateral side (as described previously) to deliver the specimen containing both thyroid lobes en bloc. Accurate orientation of the specimen is important and pertinent details of the tumor, such as intraoperative suspicion of ETE, must be communicated to a pathologist in order to ensure meaningful interpretation of histopathologic findings.

\section{Hemostasis, drains, and wound closure}

After delivery of the specimen, the wound is irrigated copiously with saline. The lower jugular, paratracheal, pretracheal, and superior mediastinal lymph node basins are inspected and palpated for any suspicious lymph-adenopathy. After total thyroidectomy, the surgeon should examine the surgical bed for any remaining thyroid tissue that may be the source of a positive RAI uptake scan and elevated thyroglobulin after surgery. The common sites of residual thyroid tissue after total thyroidectomy are the superior poles, the thyroid tissue around Berry's ligament, and the pyramidal lobe (Fig. 13).

If hypotensive anesthesia is used, the anesthesiologist should return the blood pressure to normal at this stage in order to ensure adequate hemostasis. As described previously, great caution must be exercised around the RLN and indiscriminate use of electrocautery and hemostats should be avoided.

The relevance of placing drains in the thyroid bed is debated and there is evidence to show that draining wounds does not reduce the risk for postoperative hematoma $[7,8]$. The authors prefer to drain most total thyroidectomy wounds with a Penrose drain, which generally is removed before discharge from hospital the next morning.

The wound then is closed in layers. The fascia overlying the SHMs is sutured loosely in the midline using a few interrupted chromic catgut sutures. Avoidance of a watertight closure of the strap muscles in the midline leaves a conduit between the deep and superficial neck compartments to allow shift of blood in case of expanding hematoma. If a Penrose drain is used, the drain is brought out laterally or in the midline and skin is sutured with 5-0 nylon. The wound is cleaned and smeared with Bacitracin ointment. Loose gauze fluff is used to cover the Penrose drain. After the drain is removed, patients are instructed to shower in 2 days and apply Bacitracin ointment to the suture line, which is kept exposed without dressings.

\section{Postoperative care and management of complications}

Postoperative complications are infrequent in experienced hands and best prevented by meticulous surgical technique as described previously. Early recognition and prompt initial 
management of complications are equally important to successful outcome. Apart from close supervision by the surgeon, this requires awareness and vigilance on the part of all members of the multidisciplinary team treating these patients. The most debilitating complications of thyroid surgery unquestionably are RLN injury and hypocalcemia. Other less frequent but significant complications include hematoma, seroma, ESLN injury, and wound infection.

\section{Hemorrhage and hematoma}

Neck hematoma or bleeding after thyroid surgery is rare and reportedly occurs in less than $1 \%$ of the cases [9]. The time period for development of postoperative hematoma can vary from 5 minutes to 5 days after surgery, but most hematomas occur within 6 hours after surgery. Patients undergoing thyroid surgery, therefore, are believed to benefit from close monitoring for a minimal period of 6 hours after thyroid lobectomy and overnight after total thyroidectomy [10].

Hematoma is prevented best by appropriate identification and ligation of blood vessels during surgery and by meticulous wound irrigation and hemostasis before wound closure. To identify potential bleeding vessels before wound closure, the anesthesiologist should normalize the patient's blood pressure, because hypotension and vasoconstriction may obscure potential sources of bleeding that may become manifest only after extubation. A simulated Valsalva maneuver can help identify potential venous bleeders when the anesthesiologist raises and maintains the intrapulmonary pressure. Most delayed bleeding is of venous origin and a major source rarely is apparent at wound exploration in hematomas that have occurred slowly over the postoperative course.

Rapidly expanding hematoma, alternatively, occurs most commonly from a bleeding artery. This life-threatening condition should be identified and managed as early as possible. Failure to identify impending hematoma after surgery may occur if the strap muscles are sutured tightly in the midline, allowing the hematoma to cause an increase in the central compartmental pressure with impingement of the airway. The strap muscles, therefore, should be approximated only loosely in the midline, leaving free communication between the deep and superficial neck spaces. Other causes of post-thyroidectomy hematomas include slipping of ligature on major vessels and reopening of cauterized vessels. Factors, such as elevated postoperative blood pressure and use of anticoagulant therapy, may contribute to a higher risk. Increased blood pressure immediately after extubation may be related to Valsalva maneuvers caused by bucking or vomiting and from inappropriate management of hyper-tensive patients during the early postoperative period. All these situations are preventable by close cooperation with the anesthesiologist.

Although small and stable seroma or hematoma can be monitored closely or evacuated by needle aspiration, significant or expanding hematoma of the neck should be managed surgically because of high risk for airway compromise. Symptoms, such as difficulty breathing, pressure in the neck, and voice changes with an obvious collection in the wound, require surgical intervention without delay. Patients who do not have obvious airway compromise and who have a stable hematoma should be brought to the operating room for re-exploration as early as possible after they are hemodynamically stable. In contrast, 
patients who have airway symptoms need emergency intervention at the bedside. The hematoma in such patients should be managed by opening the thyroidectomy wound at the bedside and evacuating the clotted blood. Release of central compartment tension by this maneuver usually ameliorates airway symptoms and ends the emergency. In a small percentage of patients, however, airway compromise may persist even after evacuation of the hematoma. Immediate endotracheal intubation should be performed by an experienced clinician, because these patients may develop severe airway obstruction resulting from edema of the larynx as a result of the hematoma. Emergency tracheostomy rarely may become necessary if an endotracheal airway cannot be secured. This is straightforward technically, because the trachea lies exposed in the thyroidectomy wound. All patients should be brought back to the operating room for complete wound exploration after any urgent bedside procedure. After evacuation of the hematoma, the wound should be irrigated copiously and the bleeding vessels identified and ligated. Great care must be taken to avoid injury to the RLN and PT glands, and it is prudent to avoid the use of suction directly in the wound during this phase of the operation. If emergency tracheostomy is performed, closure of the wound becomes challenging, because the tracheotomy now lies within the otherwise clean thyroidectomy wound with the potential for sepsis. The thyroidectomy wound in such instances can be closed over a Penrose drain and the tracheostomy is fashioned in the midline to isolate it from the thyroidectomy wound by suturing the edges of the tracheotomy to the cutaneous edges of the thyroidectomy wound.

The surgeon must be aware of the risk for pneumothorax and postobstructive pulmonary edema that can develop after acute airway obstruction. A chest radiograph, therefore, should be performed after exploration of hematoma in the neck to identify and treat these conditions. Patients who require re-exploration for neck hematoma should be monitored closely for at least 48 hours, because they have an increased risk for airway compromise from laryngeal edema or RLN palsy.

\section{Recurrent laryngeal nerve injury}

Temporary nerve palsy may occur in up to $5 \%$ of cases [11], whereas permanent RLN injury and vocal cord paralysis occurs in less than $2 \%$ to $3 \%$ of patients. The causes of transient nerve palsy include significant manipulation and dissection along the RLN, thermal injury, and traction injury. Alternatively, most permanent RLN injuries occur because of transaction of the nerve during surgery. Bilateral RLN injury is rare and was reported in 1 of 1000 thyroid surgeries [11]. This is an especially debilitating situation, as it creates the need for permanent tracheostomy. The surgeon should be aware of a higher risk for bilateral RLN palsy when unilateral RLN paralysis is diagnosed preoperatively, if RLN is sacrificed during the operation, or in patients undergoing reoperative thyroid surgery.

If bilateral RLN injury goes unrecognized intraoperatively, patients generally require reintubation immediately after the operation. Rarely, bilateral injury may not present for up to several hours after extubation. Recurrent failure to extubate patients clearly suggests the possibility of bilateral vocal cord paralysis. If the surgeon is confident that the RLNs are preserved during the operation, it is acceptable to treat the patient with systemic steroids and assisted endotracheal ventilation for several days. The patient then can be weaned off the 
ventilator gradually and an attempted extubation performed in the operating room. If flexible fiberoptic evaluation of the larynx clearly demonstrates an adequate airway with functioning vocal cords, no further intervention is required [12]. If, however, patients fail extubation or laryngoscopy reveals bilaterally paralyzed vocal cords with or without airway compromise, tracheostomy is indicated. Most of these patients who require tracheostomy generally have temporary RLN palsy of at least one nerve. In such cases, patients can be weaned from the tracheostomy 3 to 9 months after the operation, which is the time required for the RLNs to recover. Laryngeal electromyography may be used to assess the likelihood of future decannulation.

Patients who have unilateral RLN palsy often suffer from hoarseness, although normal voice is not infrequent in this population. Symptomatic patients who have RLN injury also may suffer from dysphagia and aspiration. As discussed previously, recovery of a nerve that is paralyzed temporarily can take several months. Symptomatic patients and those who have permanent paralysis should be considered for vocal cord medialization to improve the quality of their voice and for prevention of aspiration.

\section{Hypocalcemia and hypoparathyroidism}

Hypocalcemia secondary to iatrogenic injury to the PT glands occurs in 5\% to $25 \%$ of patients after thyroid surgery $[12,13]$. Transient hypocalcemia is more common than permanent hypocalcemia and is believed caused by impaired vascularity of the PT glands after surgery. Permanent hypocalcemia, alternatively, usually occurs because of unintentional compromise of all PT glands. A recent study from the authors' institution found that the incidence of inadvertent excision of a PT gland is approximately $8 \%$; however, removal of three or more PT glands is rare [14]. The risk for hypocalcemia increases in reoperative thyroid surgery or after paratracheal neck dissection. The postoperative measured serum calcium level also may be reported low because of surgical stress caused by inappropriate antidiuretic hormone secretion, low serum albumin level, or laboratory error. In these cases, the free or ionized calcium remains unchanged and this condition does not need correction with calcium supplementation.

Most patients who have transient hypocalcemia usually are asymptomatic. Symptomatic patients usually complain of perioral numbness, tingling, abdominal cramps, paraesthesias, or muscle spasm, especially in their upper extremities. Bedside evaluation of symptomatic patients may include tapping on the facial nerve trunk at the preauricular area, producing muscle twitches (Chvostek's sign), or occlusion of the brachial artery for 3 minutes, producing carpopedal spasm (Trousseau's sign). Failure to recognize and treat severe hypocalcemia may lead to cardiac arrhythmia and seizures.

Calcium levels are drawn routinely between 8 and 12 hours after the operation and then at 20 to 22 hours, immediately before discharge from hospital. Subsequent management is based not only on actual serum levels but also on the trend in the levels over time. Patients who have low calcium levels should be supplemented with oral calcium (400-500 mg 2 to 3 times a day), and those who have significant hypocalcemia should receive vitamin $\mathrm{D}$ therapy (0.25-0.5 mg once or twice a day) in addition. The effect of vitamin $\mathrm{D}$ on serum calcium level should be anticipated after 2 to 3 days and appropriate adjustments in the dose of 
enteral calcium may become necessary. Patients can be discharged home if normal calcium levels are achieved or if they are asymptomatic and have a rising trend of calcium levels.

Symptomatic patients and those who have severe hypocalcemia require serum calcium estimation every 3 to 6 hours and intravenous infusion of calcium $(10-20 \mathrm{~mL}$ of $10 \%$ calcium gluconate diluted in $200 \mathrm{~mL}$ of saline over 20 minutes). Intravenous calcium infusion may be continued until patient symptoms resolve. If serum calcium levels fail to normalize, serum magnesium levels should be evaluated and corrected. In cases of hypocalcemia refractory to treatment or serum calcium below $7 \mathrm{mg} / \mathrm{mL}$, an endocrinologist should be consulted.

The serum calcium level should be monitored at the first postoperative visit 7 to 10 days after discharge and supplementation should be modified accordingly. Gradual tapering of enteral calcium and calcitriol can begin if serum calcium is above $8.5 \mathrm{mg} / \mathrm{mL}$.

Supplementation should continue with appropriate adjustment of the dose for patients who maintain their serum calcium level between 7 and $8 \mathrm{mg} / \mathrm{mL}$. Patients who are severely hypocalcemic, with serum calcium below $7 \mathrm{mg} / \mathrm{mL}$, should also have an evaluation of serum parathormone.

\section{Other sequelae of thyroid surgery}

Wound infections after thyroid surgery are rare and more common in immunocompromised patients and in patients who have diabetes mellitus. Prophylactic antibiotic therapy does not decrease the rate of infection and should be used only in patients who have diabetes or valvular heart disease or in immunocompromised patients. Minor cellulitis usually is managed with antibiotics directed at Staphylococcus and Streptococcus species, whereas wound abscess should be treated with drainage and irrigation along with intravenous antibiotics therapy covering aerobic and anaerobic bacteria.

Approximately a third of patients report vocal symptoms during the early period after thyroid surgery, even in the absence of obvious evidence of nerve injury [15]. Half of these patients have persistent symptoms 3 months after the operation. Complaints of a lowered tone, vocal fatigue, and difficulty in high-pitch phonation or projection of voice should alert surgeons to the possibility of SLN injury. Videostroboscopy and laryngeal electromyography may be needed to confirm the diagnosis of SLN palsy [15]. Patients who have significant symptoms should be referred to a speech pathologist in an attempt to improve their speech.

All patients undergoing total thyroidectomy and up to a third of those after thyroid lobectomy require thyroid hormone supplementation [16]. At Memorial Sloan-Kettering Cancer Center, exogenous levothyroxine is administered, based on patient body weight early after surgery, to all patients undergoing total thyroidectomy. In patients who have WDTC requiring RAI scan or treatment, recombinant human thyrotropin administration before scanning prevents the debilitating symptoms of hypothyroidism associated with thyroid hormone withdrawal. 


\section{Large goiter and retrosternal extension}

If a retrosternal goiter is suspected, radiologic imaging using CT or MRI of the neck and superior mediastinum is required to delineate the inferior extent of the gland and its relation to the great vessels, trachea, and esophagus. Most retrosternal goiters are benign and amenable to surgical resection via the conventional cervical approach. The infrahyoid strap muscles may need to be divided on one or both sides to allow delivery of the gland. After exposure of the superior pole and capsular dissection to preserve the PT glands and RLN (described previously), gentle finger dissection is used to assess intrathoracic extension of the tumor. Retrosternal intrathoracic extension of a benign goiter generally can be mobilized safely by blunt finger dissection and delivered out of the mediastinum into the neck, as the vascular supply to the intrathoracic component almost always originates in the neck. Small vessels along the visceral fascia carefully are divided individually, only after confirming that they are devoid of neural tissue.

With large goiters, the surgeon should be aware of the possibility that the nerve may be displaced several centimeters laterally from the tracheoesophageal groove, making it difficult to identify. In these cases, the RLN may be closely adherent to the posterior capsule of the gland. In some cases, the RLN may be displaced anteriorly, and delivery of the goiter outside of the wound before identifying the RLN places the nerve at significant risk, because it may be located more superficial than its usual location and is encountered early during the dissection (Fig. 14). If the RLN anatomy is unclear, dissection alternatively may be performed in the medial-to-lateral sequence, starting with division of the thyroid isthmus. The superior pole vessels are ligated and the lobe is mobilized laterally. The RLN then can be identified at its entry to the larynx, between the cricoid cartilage and the inferior cornu of the thyroid cartilage. This is a relatively constant anatomic landmark and the course of the RLN is not subject to distortion by the goiter at this location. The RLN then is traced caudally and laterally throughout its course. If appropriate, a benign cyst may be aspirated and it may become necessary to divide a huge benign lobe to prevent stretching and injury to the RLN during delivery of the goiter.

Although the vast majority of retrosternal goiters can be excised adequately and safely via a cervical approach, thoracic exposure is required for these indications: (1) recurrent intrathoracic goiter, (2) previous mediastinal or cardiothoracic surgery, (3) previous radiation to the neck or mediastinum, (4) malignant tumor abutting the great vessels, (5) isolated intrathoracic goiter, and (6) retrotracheal goiter extending below the level of the carina [1]. Median sternotomy allows careful identification of the lower extent of the goiter and its relationship to the great vessels. Readers are referred elsewhere for a detailed description of this approach [17].

\section{Nonrecurrent laryngeal nerve}

A non-RLN should be anticipated if the nerve cannot be found inferiorly using the technique described previously. Intraoperatively, a non-RLN can be identified at its entrance to the larynx, lateral to the junction of the cricoid and lower cornu of the thyroid cartilage. This anatomic variant occurs in less than $0.5 \%$ of patients, as the RLN may bifurcate directly from the vagus above the level of the ITA [18]. This anatomic variant of the right RLN 
occurs in patients who have a retroesophageal right subclavian artery. These individuals lack the brachiocephalic artery; their right common carotid artery emerges directly from the aortic arch and the subclavian artery lies posterior to the esophagus (Fig. 15). Suspicion of this vascular anomaly should be investigated before surgery in patients who have a long history of dysphagia that cannot be explained by a large thyroid gland or other causes. The vascular anomaly associated with a nonrecurrent laryngeal nerve can be identified preoperatively using CT, MRI, or modified barium swallow test. A left-sided non-RLN is found only in association with situs inversus viscerum (ie, Kartagener's syndrome) [18] and is easier to anticipate preoperatively.

\section{Locally invasive well-differentiated thyroid cancer}

Thyroid carcinoma suspected of being locally invasive warrants preoperative evaluation with CT or MRI. These imaging modalities are helpful for evaluating the anatomic relationship of the tumor to adjacent structures to determine the involvement of the thyroid cartilage, trachea, and esophagus before surgery. Although the use of iodinated contrast generally should be avoided in imaging patients who have WDTC, locally aggressive variants tend to be less RAI avid. Contrast-enhanced CT scan, therefore, is acceptable in selected patients, because it provides important anatomic information that may be crucial to the surgeon. In cases of suspected intraluminal extension of the tumor to the trachea or esophagus, imaging should be followed by bronchoscopy and esophagoscopy, respectively. Unilateral vocal cord paralysis is an ominous sign indicative of aggressive biologic behavior of the tumor [19].

If a tumor is adherent to the perichondrium or infiltrates the laryngeal cartilage partly, the perichondrium or cartilage can be resected with the tumor. Minimal invasion of the superficial esophageal musculature may be managed adequately with submucosal resection of gross tumor while maintaining the mucosal conduit of the esophagus. This is done best after inserting a nasogastric tube at the start of the operation, facilitating surgical resection and allowing postoperative tube feeding. Minimal adherence to the tracheal cartilage can be managed with "shaving" the tumor with the tracheal cartilage to ensure total tumor resection. More aggressive surgical resection, including "sleeve resection" of the upper cervical trachea, is required if there is gross invasion of the tracheal wall with or without intraluminal extension. Similarly, total laryngectomy with or without pharyngectomy rarely becomes necessary for more locally advanced tumors. The indications and technical details of these operations are beyond the scope of this article and readers are referred to specialized surgical texts for more information $[17,20]$.

\section{Reoperative thyroid surgery}

Reoperation for recurrent cancer in the surgical bed of previously excised thyroid carcinomas is a challenging surgical procedure that carries with it various technical problems. As might be expected, dissection of the RLN in these situations can be difficult and the risk for injury and postoperative dysfunction proportionately higher.

Contrary to the usual approach for identification of the RLN close to the thyroid capsule during subcapsular dissection and preservation of the inferior PT gland, the surgical 
approach may need to be modified so that the RLN is identified inferiorly and laterally in the paratracheal groove. The RLN then can be traced cephalad but preservation of viable PT tissue becomes virtually impossible in this situation with increased risk for postoperative hypocalcemia. Paradoxically, dissection and preservation of the RLN may be easier if some amount of residual normal thyroid tissue is present, because this often indicates a previously unviolated surgical plane. Intraoperative RLN monitoring may be a useful adjunct [21-23], but it is important to recognize that preservation of anatomic integrity of the nerve does not always result in preservation of vocal cord function. Patients requiring reoperative thyroid bed surgery for recurrent tumor rather than residual normal tissue that is RAI avid should be counseled, therefore, regarding the considerably elevated risk for postoperative RLN and PT gland dysfunction.

\section{Summary}

The authors have reviewed their surgical technique for treatment of thyroid cancer and large goiters. The key features of the technique are summarized in Table 1. This technique provides a simple and versatile means of complete extracapsular thyroidectomy for lesions of the thyroid gland and minimizes postoperative complications.

\section{References}

1. Newman E, Shaha AR. Substernal goiter. J Surg Oncol. 1995; 60(3):207-212. [PubMed: 7475073]

2. Johnson JT, Wagner RL. Infection following uncontaminated head and neck surgery. Arch Otolaryngol Head Neck Surg. 1987; 113(4):368-369. [PubMed: 3814385]

3. Friedman M, LoSavio P, Ibrahim H. Superior laryngeal nerve identification and preservation in thyroidectomy. Arch Otolaryngol Head Neck Surg. 2002; 128(3):296-303. [PubMed: 11886347]

4. Skandalakis JE, Droulias C, Harlaftis N, et al. The recurrent laryngeal nerve. AmSurg. 1976; 42(9): 629-634.

5. Blumberg NA. Observations on the pyramidal lobe of the thyroid gland. S Afr Med J. 1981; 59(26): 949-950. [PubMed: 7244899]

6. Braun EM, Windisch G, Wolf G, et al. The pyramidal lobe: clinical anatomy and its importance in thyroid surgery. Surg Radiol Anat. 2007; 29(1):21-27. [PubMed: 17146601]

7. Khanna J, Mohil RS, Chintamani, et al. Is the routine drainage after surgery for thyroid necessary? A prospective randomized clinical study [ISRCTN63623153]. BMC Surg. 2005; 5:11. [PubMed: 15946379]

8. Lee SW, Choi EC, Lee YM, et al. Is lack of placement of drains after thyroidectomy with central neck dissection safe? A prospective, randomized study. Laryngoscope. 2006; 116(9):1632-1635. [PubMed: 16954994]

9. Shaha AR, Jaffe BM. Practical management of post-thyroidectomy hematoma. J Surg Oncol. 1994; 57(4):235-238. [PubMed: 7990478]

10. Lacoste L, Gineste D, Karayan J, et al. Airway complications in thyroid surgery. Ann Otol Rhinol Laryngol. 1993; 102(6):441-446. [PubMed: 8512271]

11. Muller PE, Jakoby R, Heinert G, et al. Surgery for recurrent goitre: its complications and their risk factors. Eur J Surg. 2001; 167(11):816-821. [PubMed: 11848234]

12. Fewins J, Simpson CB, Miller FR. Complications of thyroid and parathyroid surgery. Otolaryngol Clin North Am. 2003; 36(1):189-206. [PubMed: 12803016]

13. Shemen LJ, Strong EW. Complications after total thyroidectomy. Otolaryngol Head Neck Surg. 1989; 101(4):472-475. [PubMed: 2508025]

14. Lin DT, Patel SG, Shaha AR, et al. Incidence of inadvertent parathyroid removal during thyroidectomy. Laryngoscope. 2002; 112(4):608-611. [PubMed: 12150510] 
15. Stojadinovic A, Shaha AR, Orlikoff RF, et al. Prospective functional voice assessment in patients undergoing thyroid surgery. Ann Surg. 2002; 236(6):823-832. [PubMed: 12454521]

16. McHenry CR, Slusarczyk SJ. Hypothyroidisim following hemithyroidectomy: incidence, risk factors, and management. Surgery. 2000; 128(6):994-998. [PubMed: 11114634]

17. Shah, JP.; Patel, SG. Head and neck surgery and oncology. 3rd edition. St. Louis (MO): Mosby; 2003.

18. Henry JF, Audiffret J, Denizot A, et al. The nonrecurrent inferior laryngeal nerve: review of 33 cases, including two on the left side. Surgery. 1988; 104(6):977-984. [PubMed: 3057672]

19. Randolph GW, Kamani D. The importance of preoperative laryngoscopy in patients undergoing thyroidectomy: voice, vocal cord function, and the preoperative detection of invasive thyroid malignancy. Surgery. 2006; 139(3):357-362. [PubMed: 16546500]

20. Grillo HC, Zannini P. Resectional management of airway invasion by thyroid carcinoma. Ann Thorac Surg. 1986; 42(3):287-298. [PubMed: 3753077]

21. Farrag TY, Agrawal N, Sheth S, et al. Algorithm for safe and effective reoperative thyroid bed surgery for recurrent/persistent papillary thyroid carcinoma. Head Neck. 2007; 29:1069-1074. [PubMed: 17563908]

22. Chan WF, Lang BH, Lo CY. The role of intraoperative neuromonitoring of recurrent laryngeal nerve during thyroidectomy: a comparative study on 1000 nerves at risk. Surgery. 2006; 140(6): 866-872. [PubMed: 17188132]

23. Yarbrough DE, Thompson GB, Kasperbauer JL, et al. Intraoperative electromyographic monitoring of the recurrent laryngeal nerve in reoperative thyroid and parathyroid surgery. Surgery. 2004; 136(6):1107-1115. [PubMed: 15657563] 


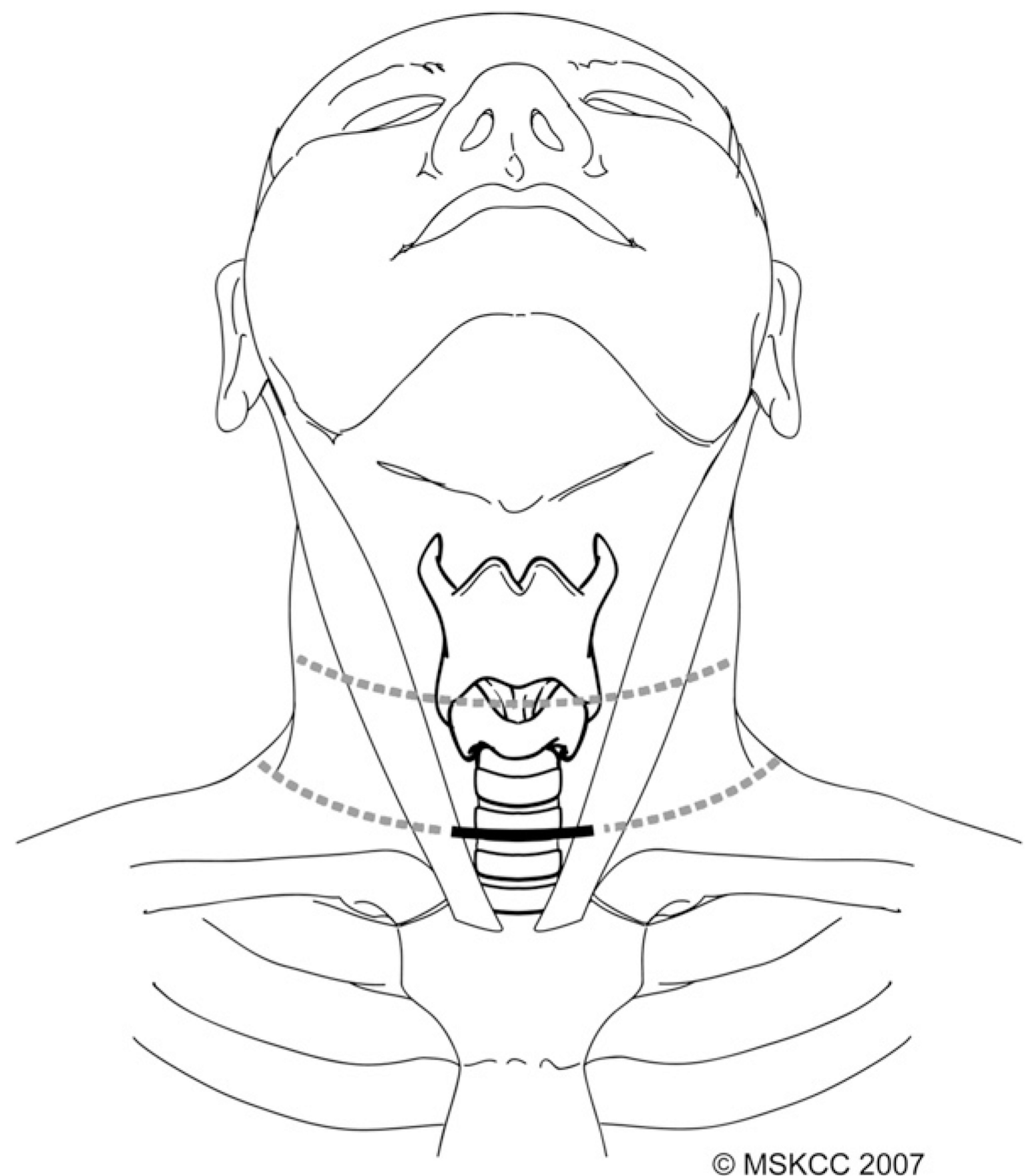

Fig. 1.

A transverse skin crease in the lower neck is chosen most commonly for the skin incision (bold line). This incision can be extended laterally if neck dissection is required or, alternatively, a higher skin crease can be selected (dotted lines). (Courtesy of the Memorial Sloan-Kettering Cancer Center, New York, NY; with permission.) 


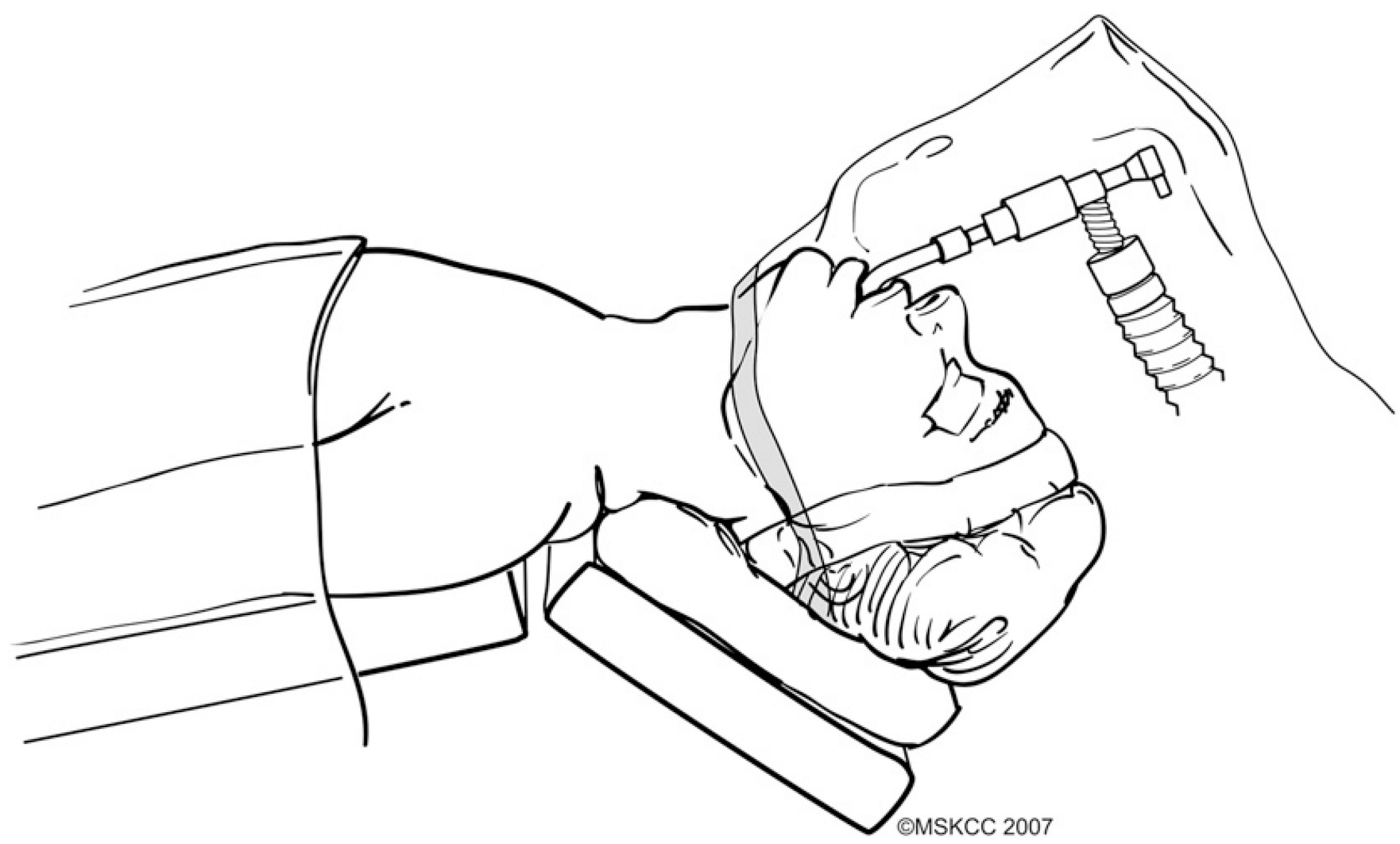

Fig. 2.

The patient is positioned supine on the operating table, which is maintained in a reverse Trendelenburg position with the neck extended. A transparent plastic drape allows continuous monitoring of the endotracheal tube and anesthesia circuit. (Courtesy of the Memorial Sloan-Kettering Cancer Center, New York, NY; with permission.) 


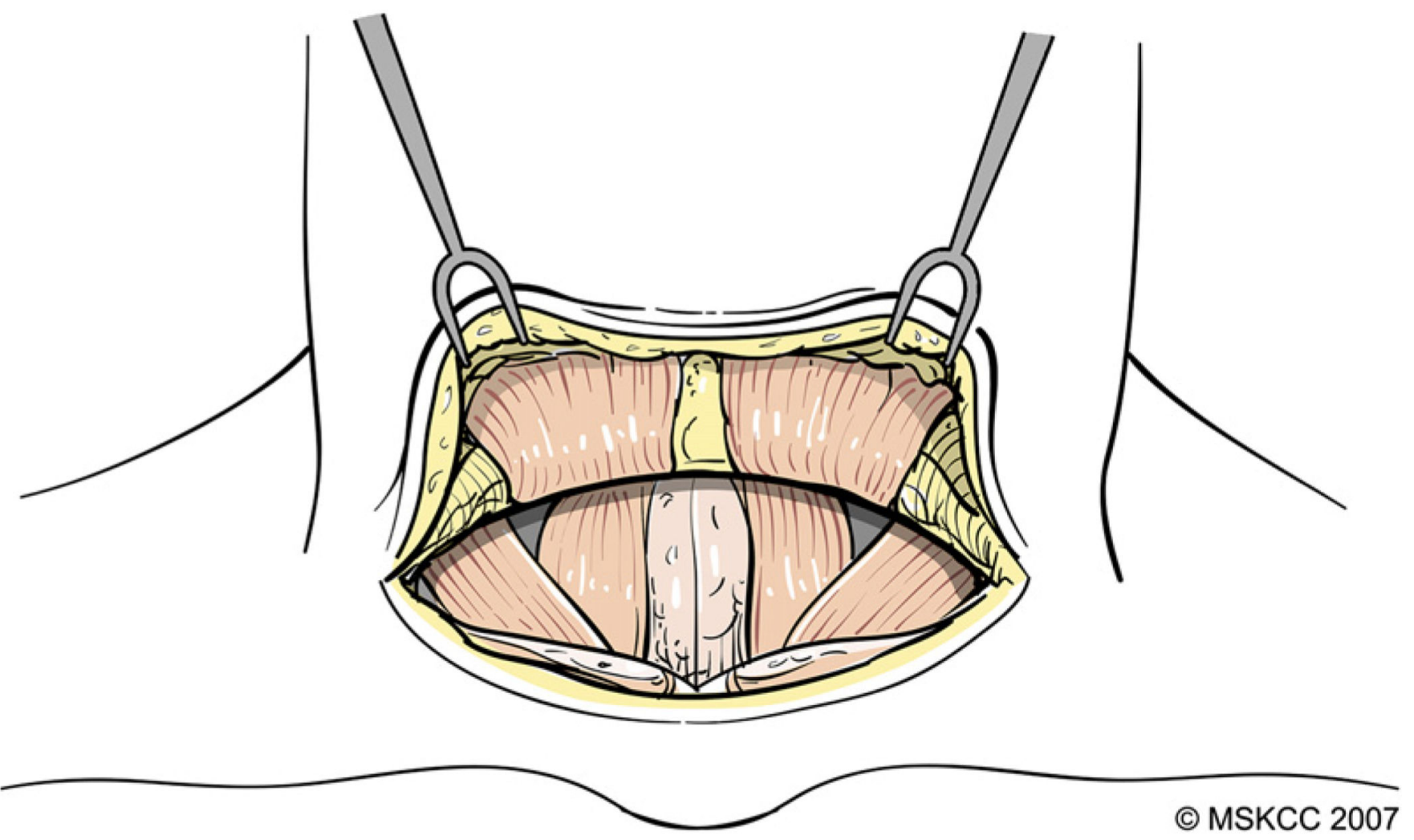

Fig. 3.

The platysma muscle is incised and cutaneous flaps are developed in the subplatysmal plane.

(Courtesy of the Memorial Sloan-Kettering Cancer Center, New York, NY; with permission.) 

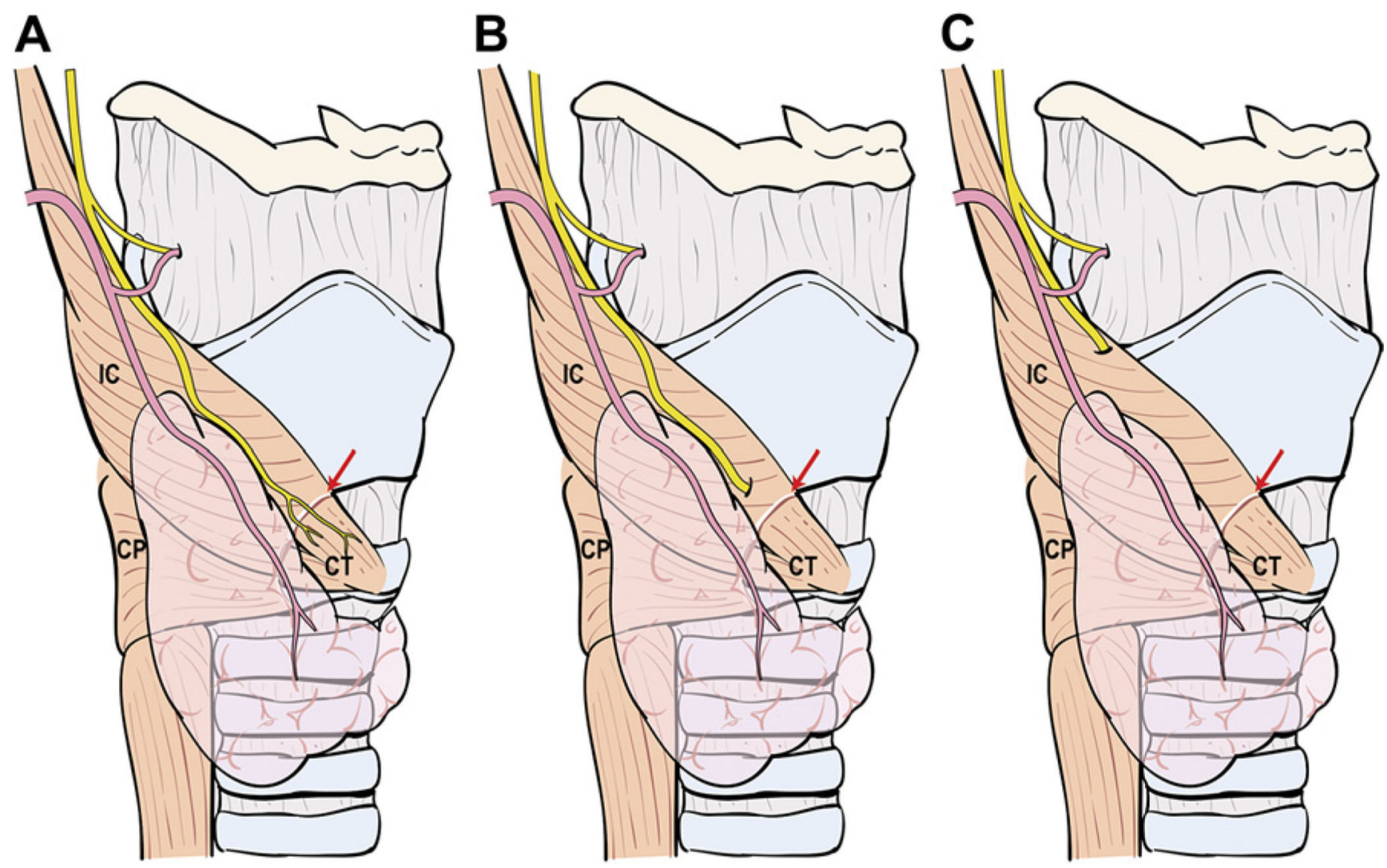

Fig. 4.

Variations in the anatomic relationship of the main trunk of the ESLN to the inferior constrictor (IC) muscle and superior thyroid pedicle. (A) The ESLN descends superficial to the IC muscle along the superior thyroid vessels so that it is visible in its entire course before innervating the cricothyroid (CT) muscle. (B) The ESLN pierces the IC muscle approximately $1 \mathrm{~cm}$ above the cricothyroid membrane (red arrow) so that only its upper portion is at risk for injury. $(C)$ The ESLN runs deep to the IC muscle and, therefore, is protected from unintended injury during dissection in the vicinity of the superior thyroid pole. The cricopharyngeus muscle is marked CP. 


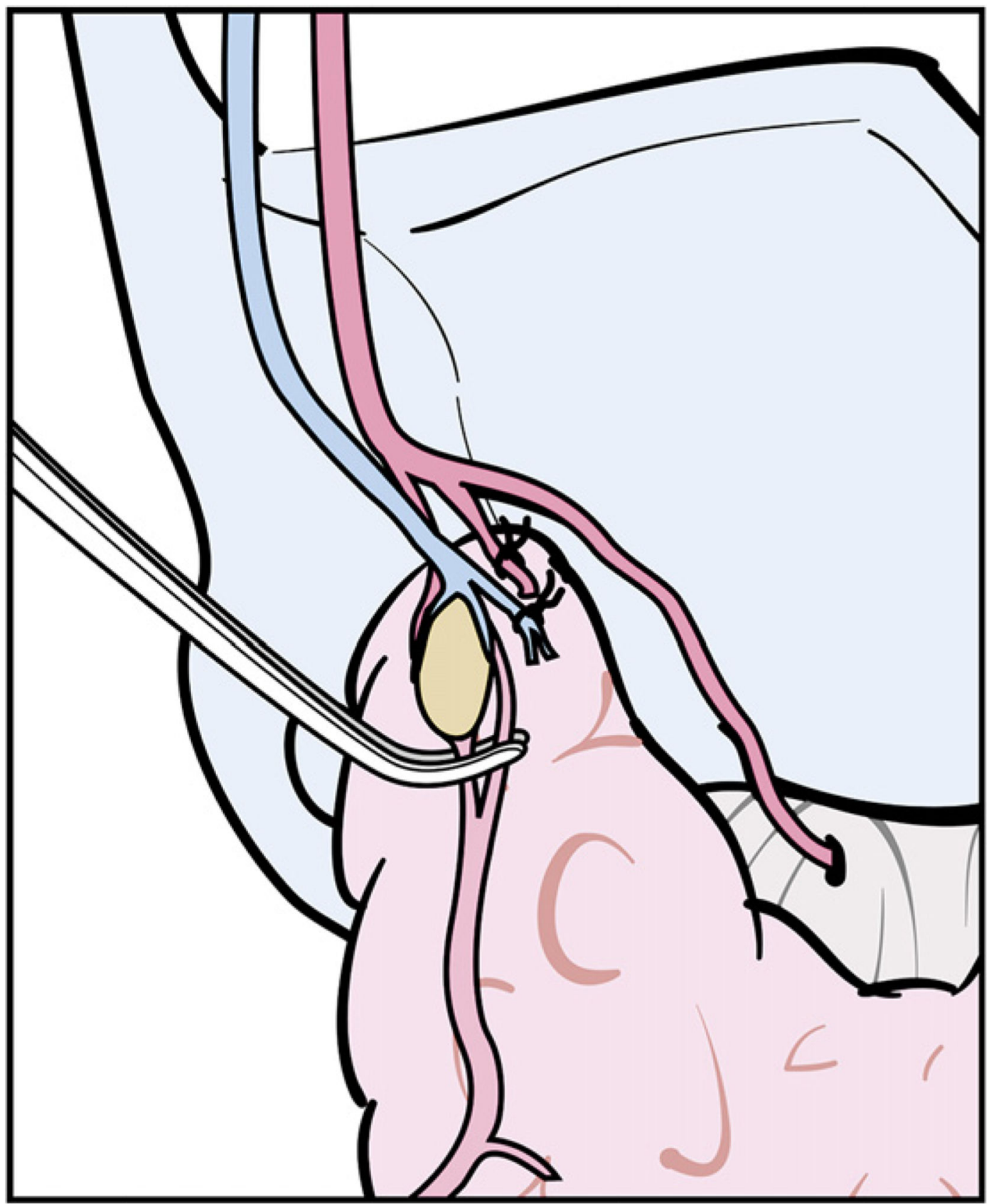

(C) MSKCC 2007

Fig. 5.

The technique of individual vessel ligation allows the surgeon to delineate the thyroid parenchymal tissue at the superior pole from its surrounding structures, minimizing risk for injury to the ESLN. (Courtesy of the Memorial Sloan-Kettering Cancer Center, New York, NY; with permission.) 


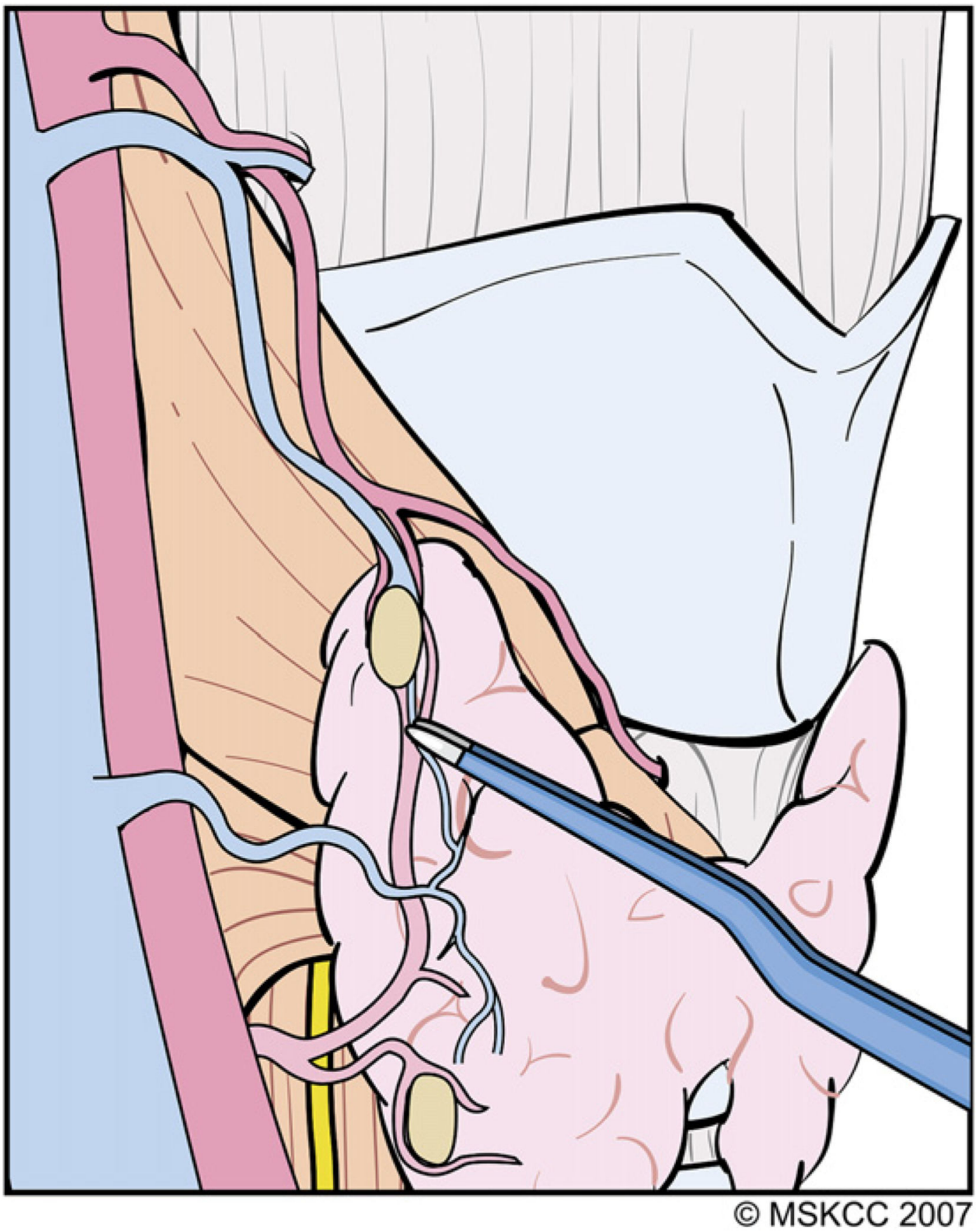

Fig. 6.

As the superior pole tissue drops down away from the ESLN, the remaining small blood vessels, especially those in the vicinity of the superior PT gland, can be cauterized safely with fine-tipped bipolar electrocautery. (Courtesy of the Memorial Sloan-Kettering Cancer Center, New York, NY; with permission.) 


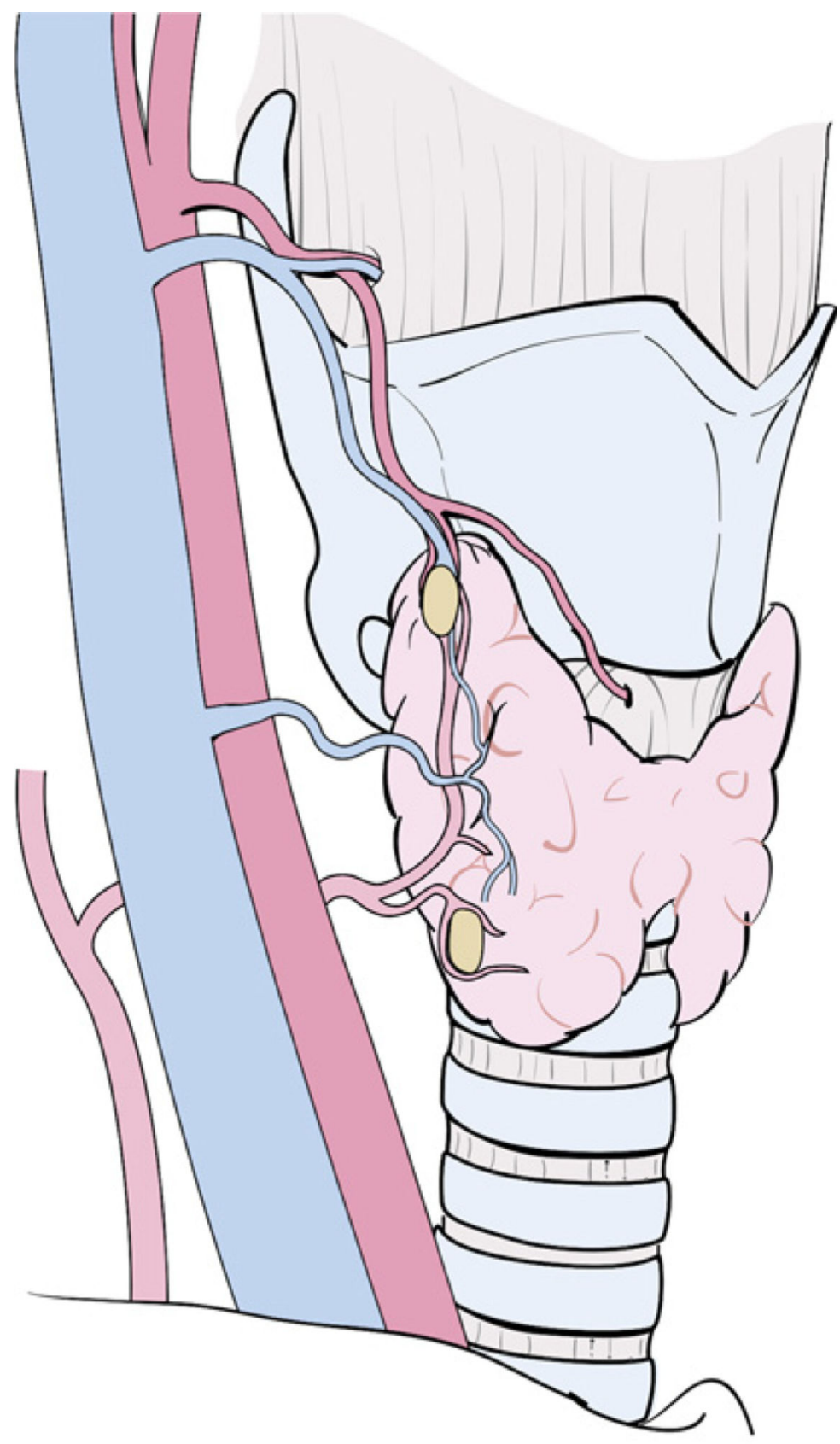

(C) MSKCC 2007

Fig. 7.

The PT glands receive their blood supply from branches of the ITA and, less frequently, from the superior thyroid artery. (Courtesy of the Memorial Sloan-Kettering Cancer Center, New York, NY; with permission.) 


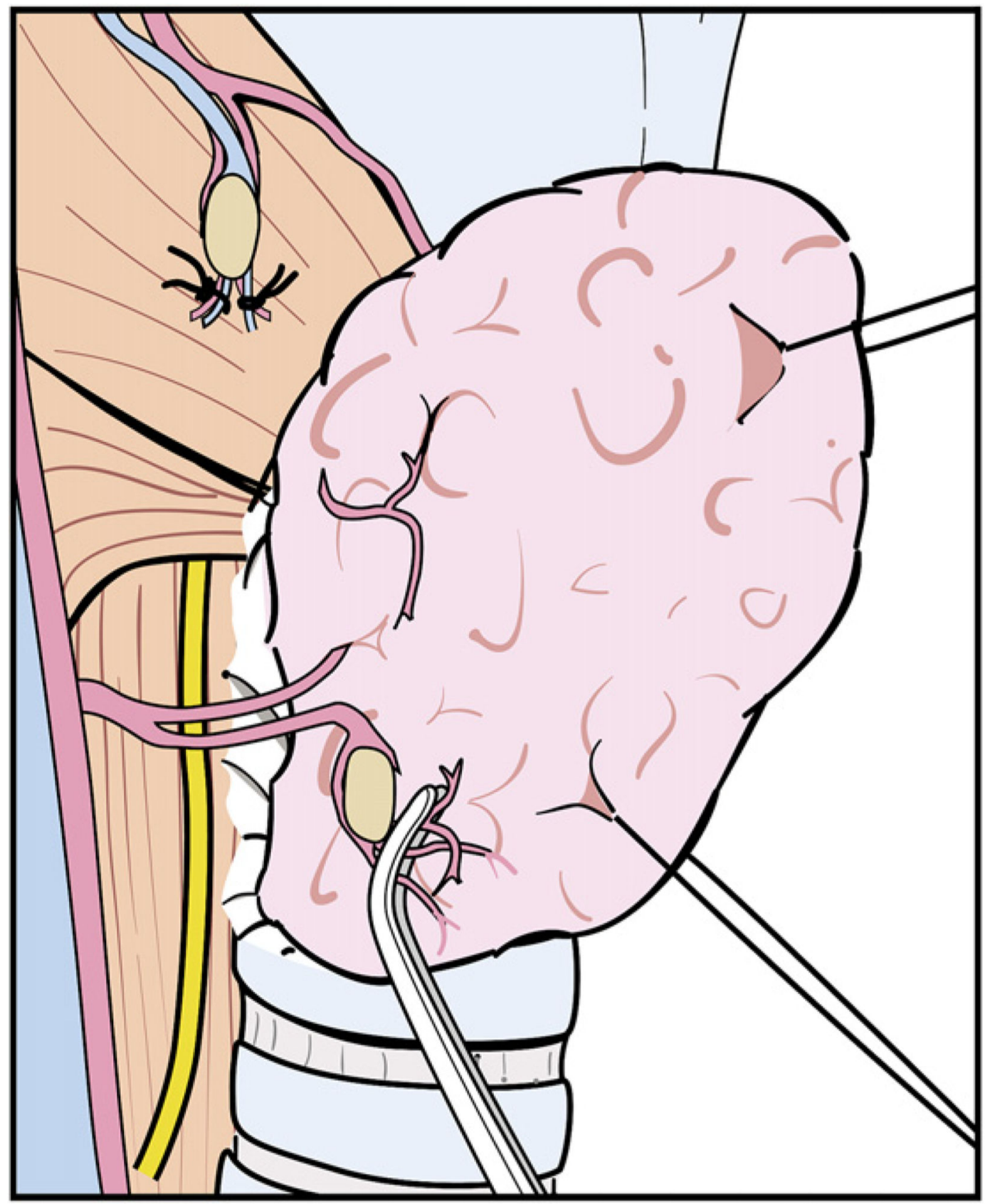

(C) MSKCC 2007

Fig. 8.

The terminal branches of the ITA that are encountered distal to the PT gland after they have supplied these glands are divided and ligated carefully. As the areolar tissue around the thyroid capsule is peeled away along with the PT gland, the RLN comes into view.

(Courtesy of the Memorial Sloan-Kettering Cancer Center, New York, NY; with permission.) 

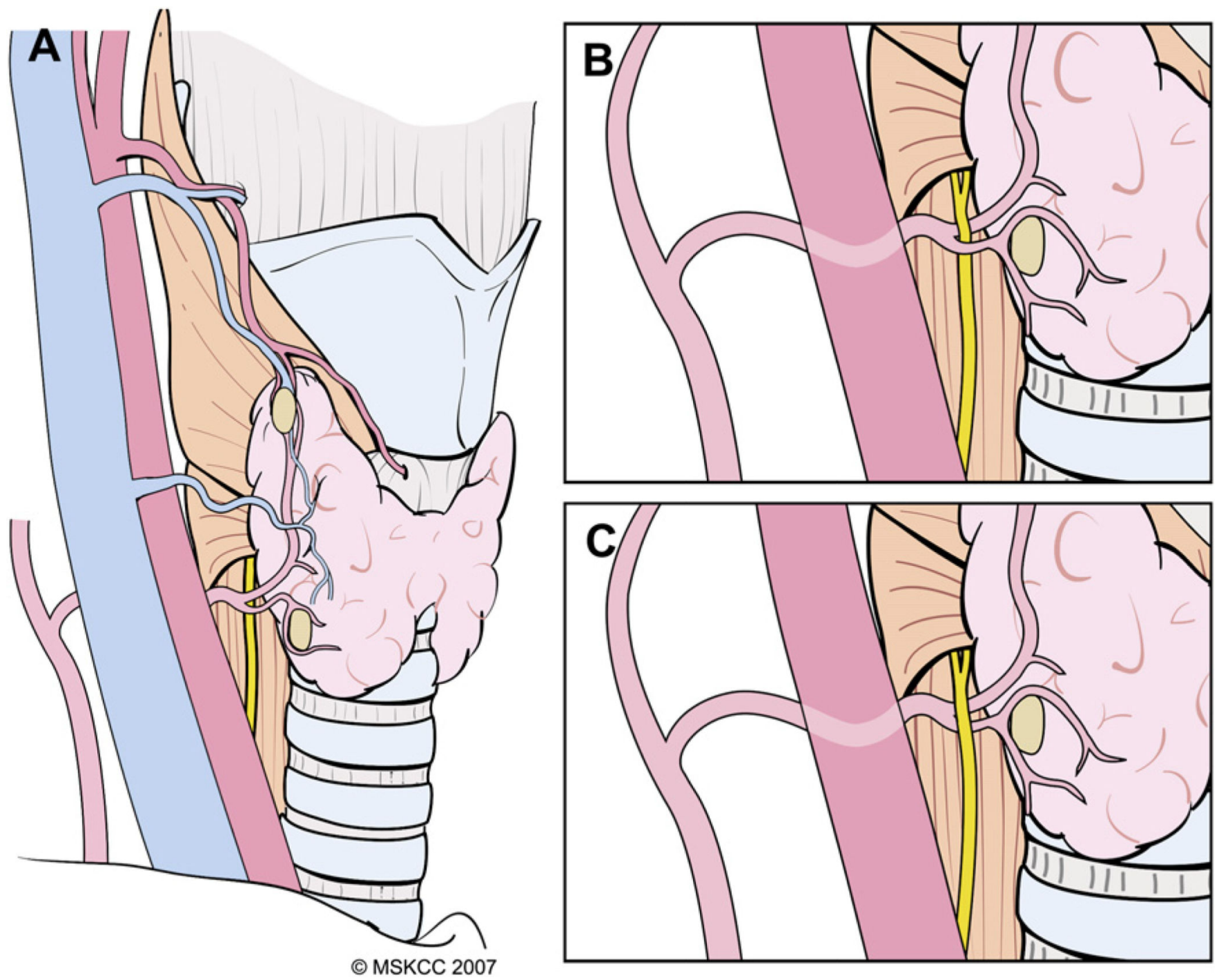

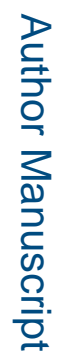

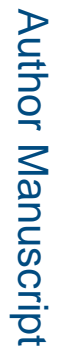

Fig. 9.

Variations in the relationship of the RLN to the ITA and its branches. Most commonly, the RLN courses deep to the inferior thyroid artery and its branches $(A)$, but it may lie between the branches $(B)$ or anterior $(C)$ to the artery. (Courtesy of the Memorial Sloan-Kettering Cancer Center, New York, NY.) 

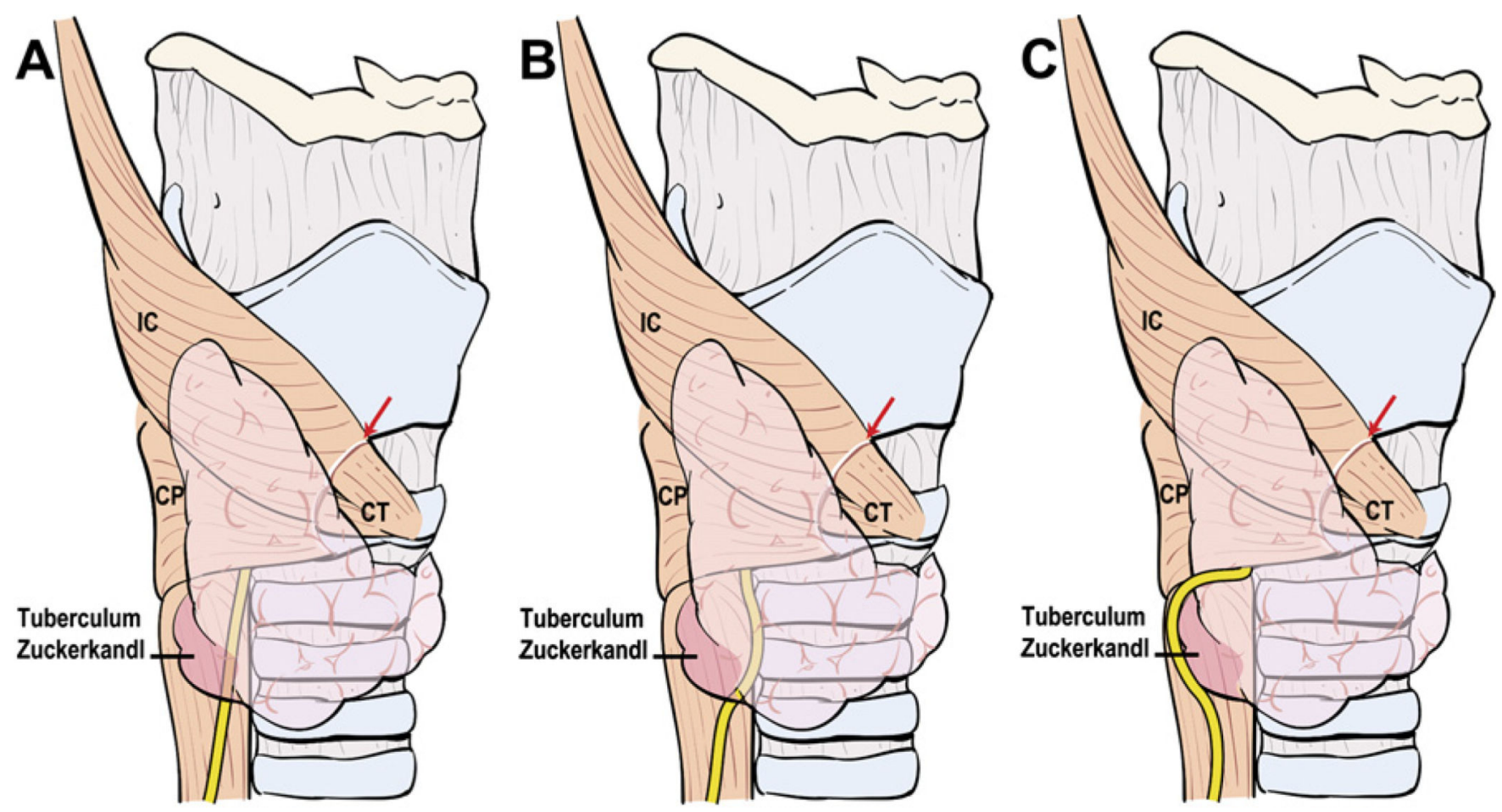

Fig. 10.

Variations in anatomic relationships of the RLN to the tuberculum Zuckerkandl. The RLN generally courses deep to the tuberculum Zuckerkandl and superficial to the lateral border of the trachea $(A)$ but it may run medial to it $(B)$. Nodular enlargement of thyroid tissue in the location of the tuberculum $(C)$ may displace the RLN laterally around it, placing the nerve at risk for injury if this variation is not recognized. IC, inferior constrictor muscle; CP, cricopharyngeus muscle; CT, cricothyroid muscle. 

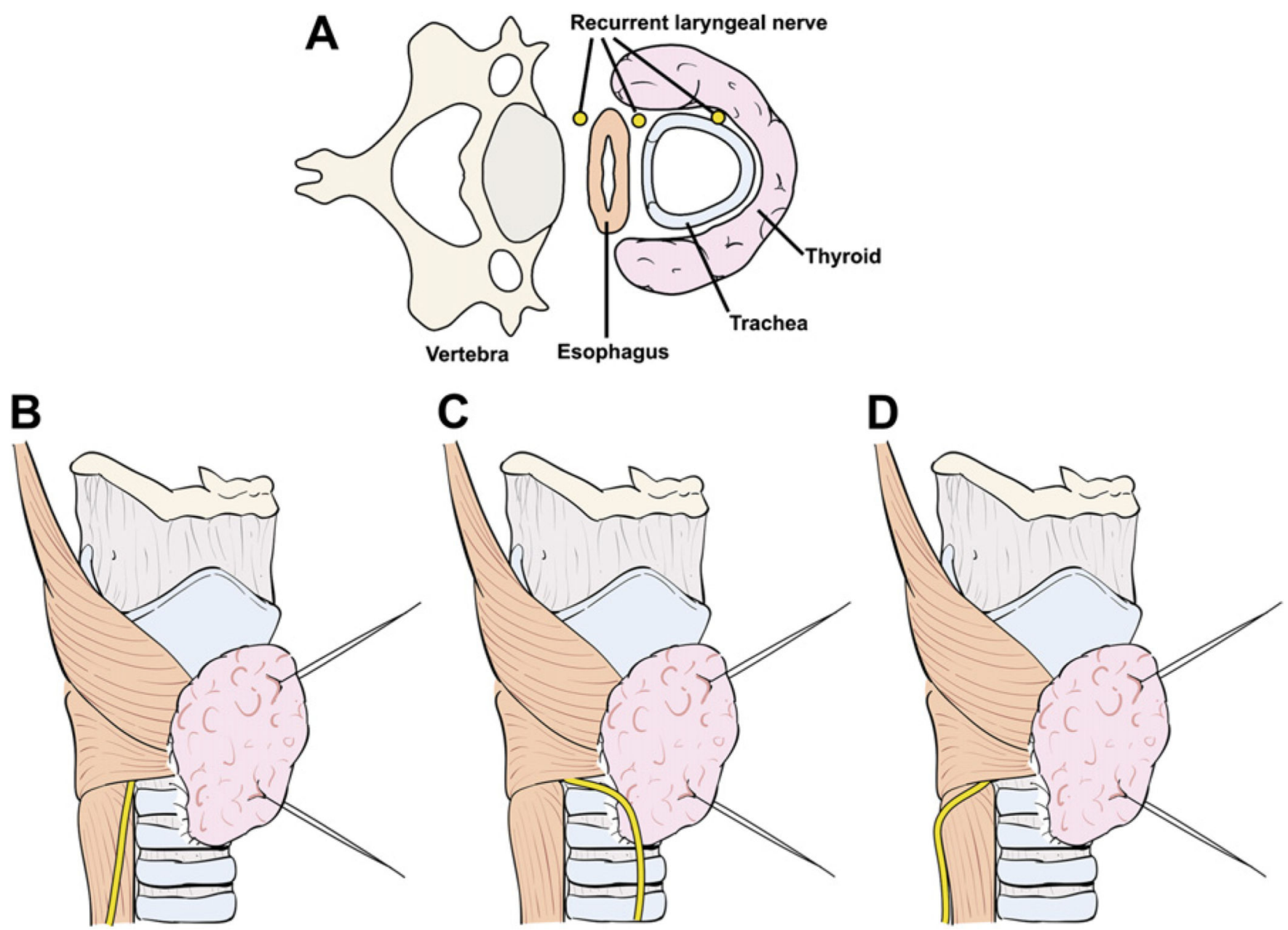

Fig. 11.

Anatomic relationship of the RLN to the tracheoesophageal groove $(A)$. Most commonly, the RLN courses along the tracheoesophageal groove $(B)$ and passes deep to Berry's ligament. Variations include an RLN that ascends anterior to the tracheoesophageal groove $(C)$ or along the paraesophageal line posterior to the tracheoesophageal groove $(D)$. 


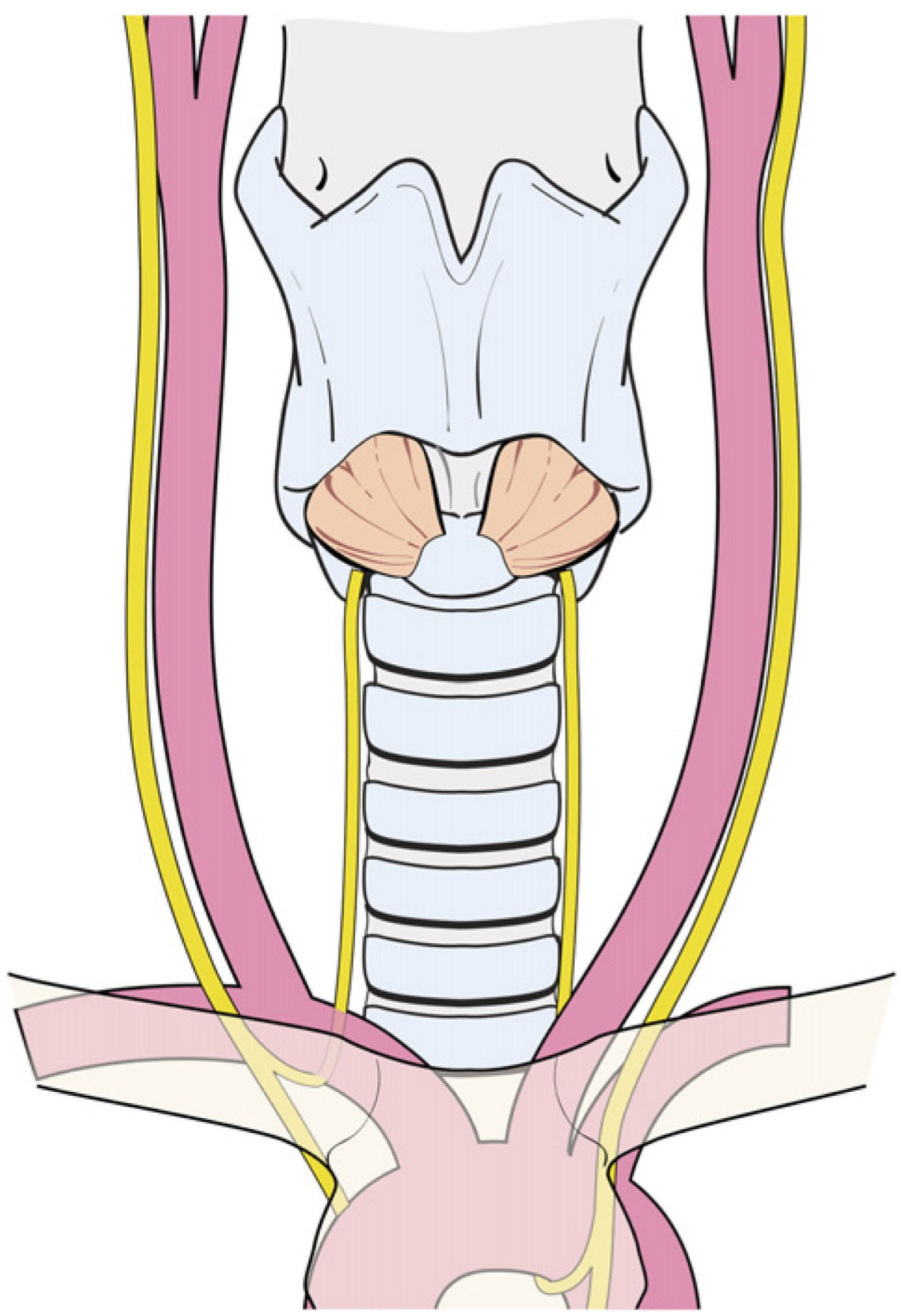

Fig. 12.

The left RLN crosses anterior to the aortic arch, loops under it, and ascends in a relatively constant position along the tracheoesophageal groove. 


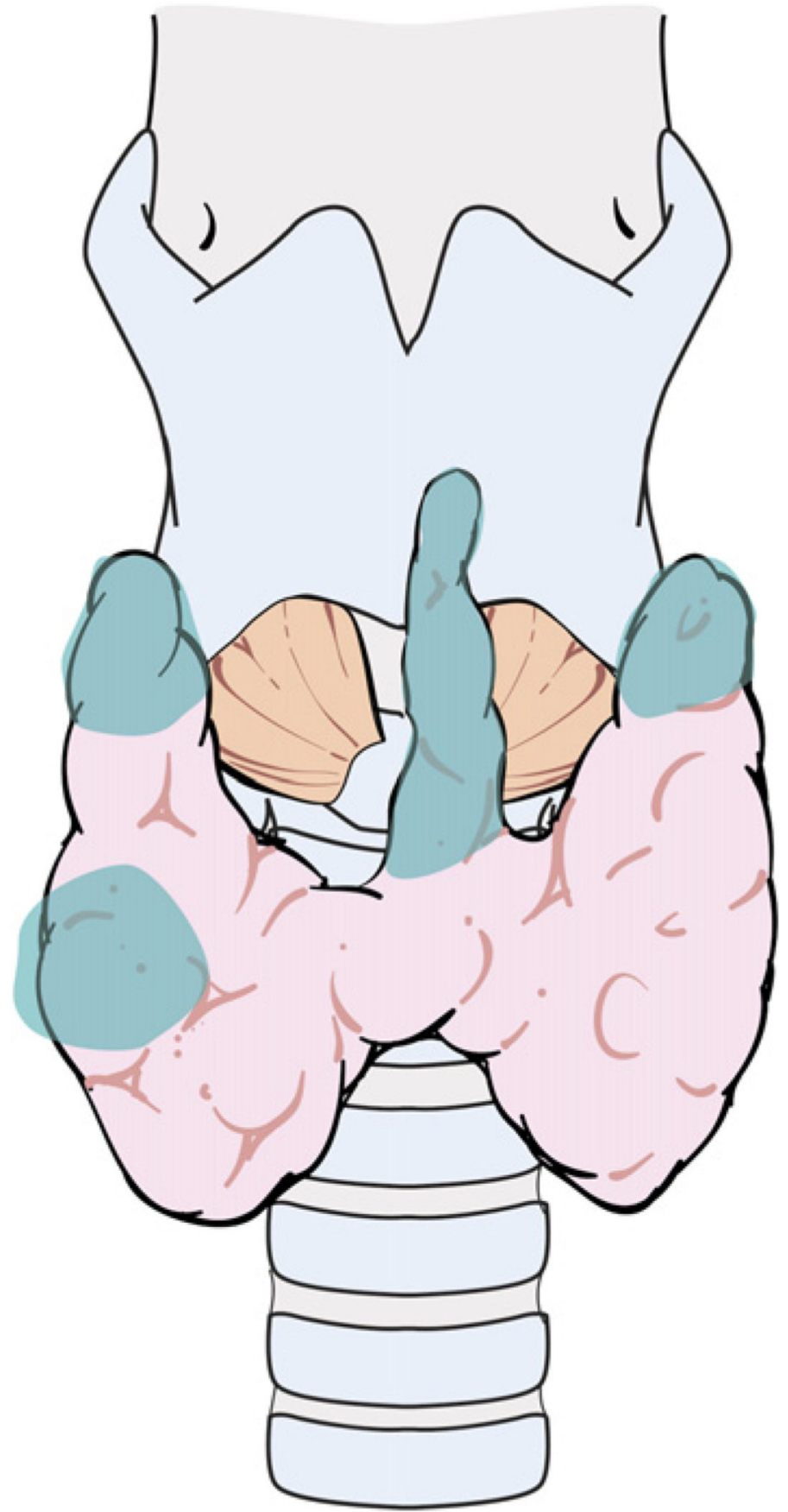

Fig. 13.

The common sites of residual thyroid tissue after total thyroidectomy are the superior poles, the thyroid tissue around Berry's ligament, and the pyramidal lobe. 


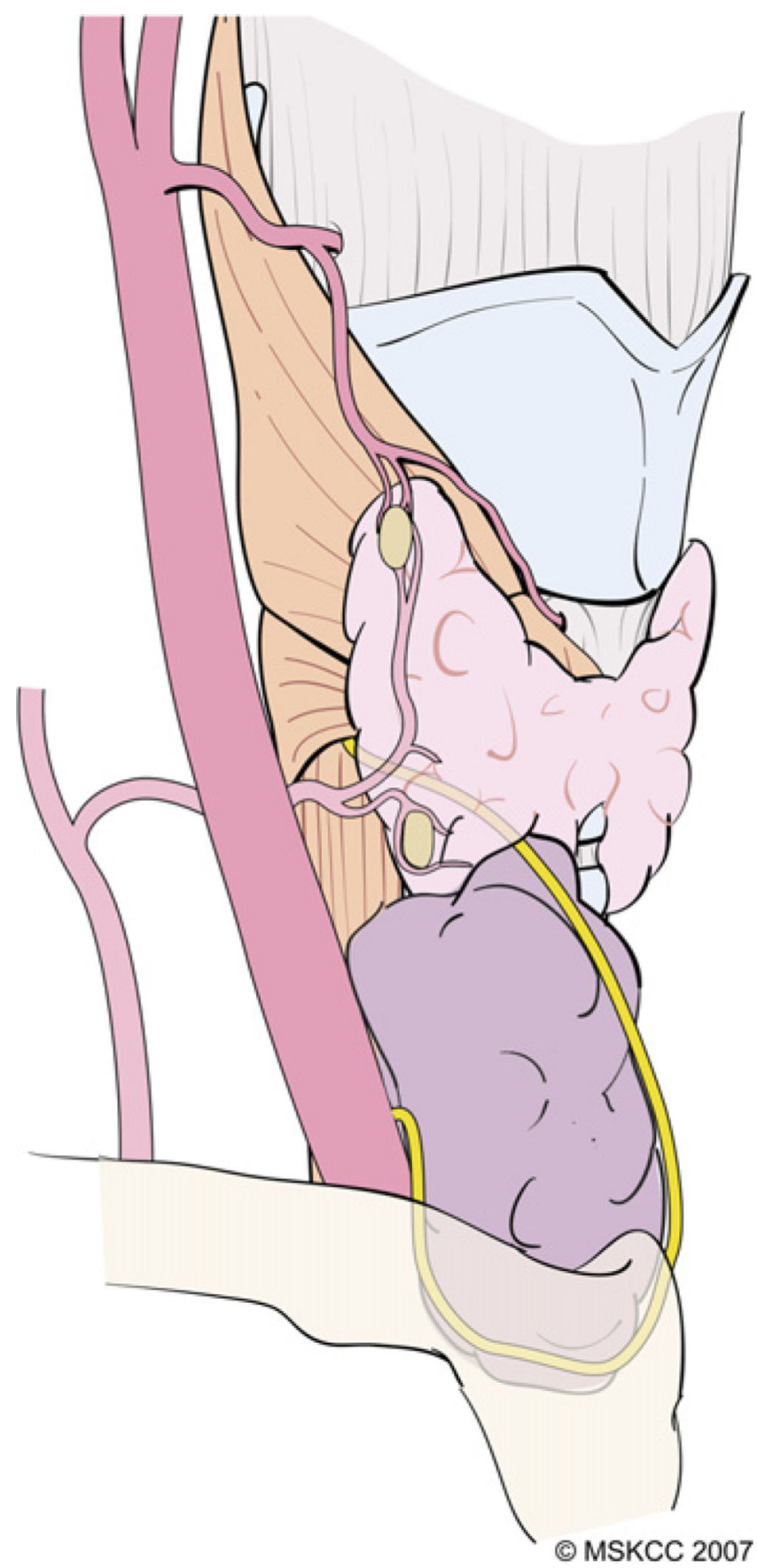

Fig. 14.

The RLN rarely may be displaced anteriorly if a nodule arises from a posterior location on the lower pole. If such a nodule extends retrosternally, attempts at delivery of the goiter outside of the wound before identifying the RLN places the nerve at significant risk, as it may be located more superficial than its usual location and is encountered early during the dissection. (Courtesy of the Memorial Sloan-Kettering Cancer Center, New York, NY; with permission.) 


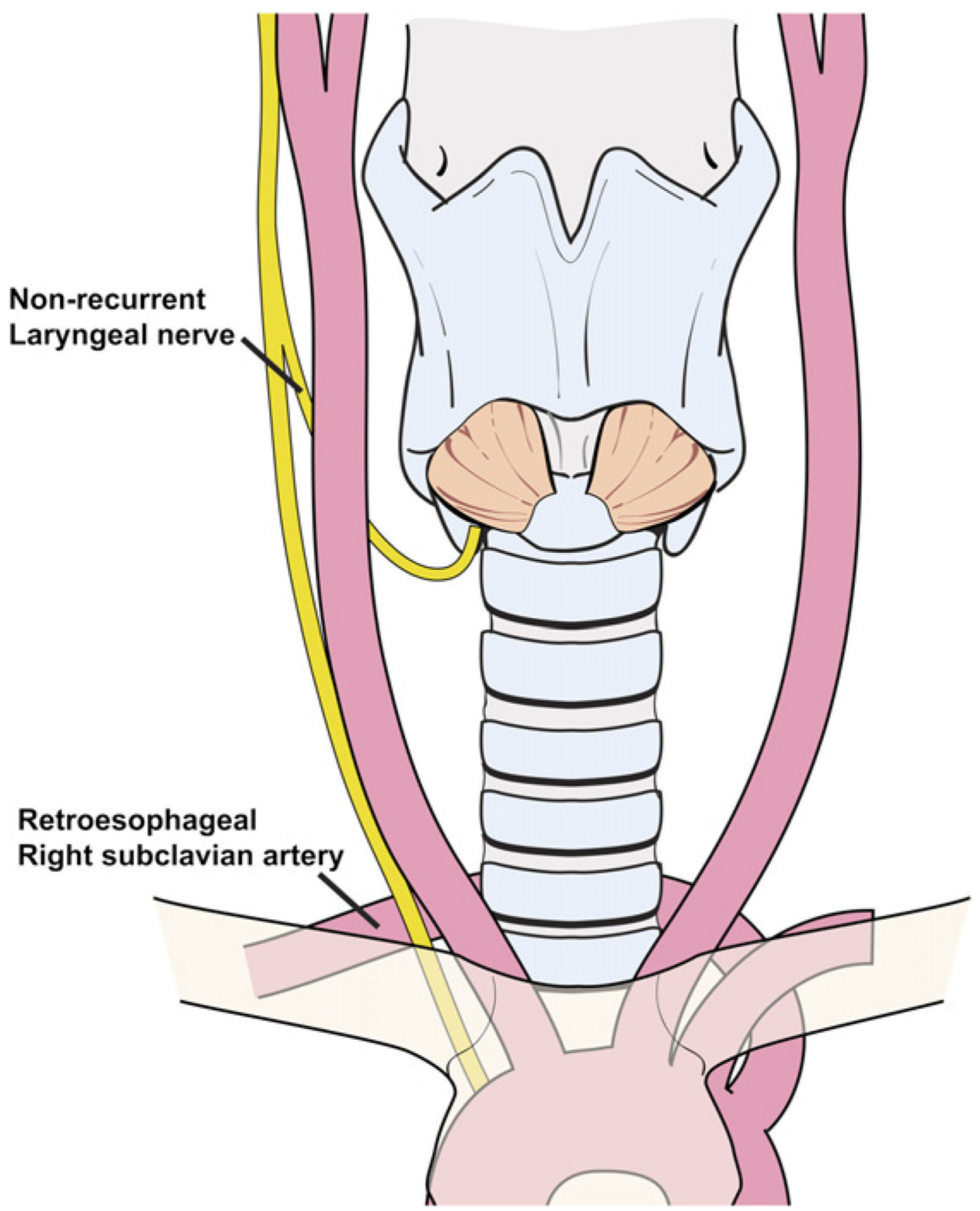

Fig. 15.

A nonrecurrent right laryngeal nerve occurs in patients who have a retroesophageal right subclavian artery. 


\section{Table 1}

\section{Summary of surgical technique for thyroidectomy}

\begin{tabular}{|c|c|c|}
\hline Positioning and draping & • & $\begin{array}{l}\text { Reverse Trendelenburg with neck extended } \\
\text { Transparent plastic drape helps monitor endotracheal tube and anesthesia circuit }\end{array}$ \\
\hline Surgical incision & • & Vary location and length depending on body habitus and exposure required \\
\hline Cutaneous flaps & • & Elevate in the subplatysmal plane \\
\hline Management of strap muscles & • & $\begin{array}{l}\text { Retraction without division for accessible superior pole } \\
\text { Division at superior attachment for inaccessible superior pole } \\
\text { Segmental muscle resection if suspected or gross invasion by tumor } \\
\text { Division of both strap muscles for huge retrosternal goiter }\end{array}$ \\
\hline $\begin{array}{l}\text { Superior pole dissection and } \\
\text { preservation } \\
\text { of ESLN }\end{array}$ & $\bullet$ & $\begin{array}{l}\text { Ligate individual branches of the superior thyroid artery anterior to the pole to avoid injury to } \\
\text { parathyroid and ESLN } \\
\text { Avoid mass ligature of superior pedicle and excise all thyroid tissue }\end{array}$ \\
\hline $\begin{array}{l}\text { Capsular dissection and } \\
\text { preservation } \\
\text { of PT glands }\end{array}$ & • & $\begin{array}{l}\text { Use fine-tipped clamps for dissection } \\
\text { Ligate branches of ITA on the thyroid capsule distal to the PT gland to ensure preservation of } \\
\text { the blood supply to the PT glands }\end{array}$ \\
\hline Identification of RLN & $\bullet$ & $\begin{array}{l}\text { Avoid lateral dissection for identification of RLN in paratracheal groove as the initial step } \\
\text { Recognize variations in normal anatomy relative to tracheoesophageal groove, branches of the } \\
\text { ITA, tuberculum Zuckerkandl, and Berry's ligament } \\
\text { Anticipate distortions in normal relationship of RLN to adjoining structures due to tumorand } \\
\text { Berry's ligament }\end{array}$ \\
\hline $\begin{array}{l}\text { Hemostasis, drains, and wound } \\
\text { closure }\end{array}$ & • & $\begin{array}{l}\text { Avoid suction drain } \\
\text { Layered wound closure }\end{array}$ \\
\hline Postoperative care & • & $\begin{array}{l}\text { Monitor airway } \\
\text { Monitor neck wound for hematoma } \\
\text { Monitor blood work for hypocalcemia }\end{array}$ \\
\hline
\end{tabular}

